\title{
Milk proteins as mastitis markers in dairy ruminants - a systematic review
}

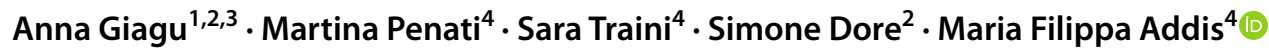

Received: 26 September 2021 / Accepted: 8 February 2022 / Published online: 23 February 2022

(c) The Author(s) 2022

\begin{abstract}
Mastitis is one of the most impacting diseases in dairy farming, and its sensitive and specific detection is therefore of the greatest importance. The clinical evaluation of udder and mammary secretions is typically combined with the milk Somatic Cell Count (SCC) and often accompanied by its bacteriological culture to identify the causative microorganism. In a constant search for improvement, several non-enzymatic milk proteins, including milk amyloid A (M-SAA), haptoglobin (HP), cathelicidin (CATH), and lactoferrin (LF), have been investigated as alternative biomarkers of mastitis for their relationship with mammary gland inflammation, and immunoassay techniques have been developed for detection with varying degrees of success. To provide a general overview of their implementation in the different dairy species, we carried out a systematic review of the scientific literature using the PRISMA (Preferred Reporting Items for Systematic Reviews and Meta-Analysis) guidelines. Our review question falls within the type "Diagnostic test accuracy questions" and aims at answering the diagnostic question: "Which are the diagnostic performances of mastitis protein biomarkers investigated by immunoassays in ruminant milk?". Based on 13 keywords combined into 42 searches, 523 manuscripts were extracted from three scientific databases. Of these, 33 passed the duplicate removal, title, abstract, and full-text screening for conformity to the review question and document type: $78.8 \%$ investigated cows, $12.1 \%$ sheep, $9.1 \%$ goats, and $6.1 \%$ buffaloes (some included more than one dairy species). The most frequently mentioned protein was M-SAA (48.5\%), followed by HP (27.3\%), CATH (24.2\%) and LF (21.2\%). However, the large amount of heterogeneity among studies in terms of animal selection criteria (45.5\%), index test (87.9\%), and standard reference test (27.3\%) resulted in a collection of data not amenable to meta-analysis, a common finding illustrating how important it is for case definitions and other criteria to be standardized between studies. Therefore, results are presented according to the SWiM (Synthesis Without Meta-analysis) guidelines. We summarize the main findings reported in the 33 selected articles for the different markers and report their results in form of comparative tables including sample selection criteria, marker values, and diagnostic performances, where available. Finally, we report the study limitations and bias assessment findings.
\end{abstract}

Keywords immunoassay $\cdot$ intramammary infection $\cdot$ amyloid A $\cdot$ haptoglobin $\cdot$ cathelicidin

\section{Introduction}

Maria Filippa Addis

filippa.addis@unimi.it

1 Dipartimento di Medicina Veterinaria, Università di Sassari, Sassari, Italy

2 Istituto Zooprofilattico Sperimentale della Sardegna, Centro di Referenza Nazionale per le Mastopatie degli Ovini e dei Caprini, Sassari, Italy

3 ARES Sardegna, ASL, Nuoro, Italy

4 Dipartimento di Medicina Veterinaria e Scienze Animali, Università di Milano, Lodi, Italy
As a critical factor affecting milk yield and quality, mastitis represents the most relevant health problem in dairy ruminants worldwide (Ruegg 2017). According to the National Mastitis Council (NMC) mastitis is defined as "an inflammation of one or more quarters/halves of the mammary gland, almost always caused by an infecting microorganism" (Lopez-Benavides et al. 2012). Whereas clinical mastitis can be diagnosed by examination of the udder and of the milk for visible abnormalities, identifying subclinical mastitis is more challenging (Menzies and Ramanoon 2001; Oliver et al. 2004). In animals with subclinical mastitis, the 
diagnosis is mainly performed on the milk through indirect methods such as the Somatic Cell Count (SCC) (Bergonier et al. 2003; Persson and Olofsson 2011) or its field version, the California Mastitis Test (CMT) (Kelly et al. 2018). Being typically caused by an intramammary infection (IMI) (Ezzat Alnakip et al. 2014), the disease is also investigated through direct methods such as the bacteriological culture (BC) (Contreras et al. 2007) or molecular assays (i.e., PCR) (Chakraborty et al. 2019). The indirect screening approaches rely mainly on the principle that the udder microenvironment changes during the inflammatory process, with an increase in the concentration of immune cells and immune mediators (Hughes and Watson 2018). Polymorphonuclear neutrophils (PMNs) are the prevalent immune cells in the acute phase of mastitis; therefore, SCC and CMT perform well as diagnostic tools because of their indirect relationship to the presence of PMNs (Leitner et al. 2000; Sordillo and Streicher 2002). However, these tests may lack specificity (Rossi et al. 2018), especially in small ruminants (Souza et al. 2012). On the other hand, BC lacks sensitivity (Chakraborty et al. 2019), and it is hardly applicable as a mastitis screening tool given its requirements in terms of time, labor, and cost. Clinical examination, SCC, CMT, and BC, should be used in combination for increasing diagnostic performance (Lam et al. 2009; Chakraborty et al. 2019); however, a universally accepted specific diagnostic algorithm or protocol is not yet available.

During mammary gland inflammation, numerous antibacterial and immune defense proteins, including Acute Phase Proteins (APPs), lactoferrin (LF), cathelicidins (CATH), cytokines, chemokines, and growth factors, are released in the milk and can potentially serve as "mastitis markers" (Smolenski et al. 2011; Thomas et al. 2015). Accordingly, their implementation as alternative/integrative diagnostic tools has been the subject of several studies during the last decades (Viguier et al. 2009). Many of them focused on discovering new biomarkers for implementing diagnostic tools with improved sensitivity and specificity when compared to the currently available assays. For inflammation-related proteins devoid of intrinsic enzymatic activity, the measurement methods are typically immunoassays employing highly specific antibodies (Viguier et al. 2009). Adding to the possibility of increased diagnostic performances, the integration of traditional diagnostic approaches with immunoassays measuring mastitis marker proteins might bring additional benefits, including the ability to work efficiently on frozen samples, the high analytical throughput, the relatively low analytical costs, and the minimal requirements for dedicated personnel training, specialized or expensive instrumentations (Addis et al. 2016a).

A group of widely investigated potential biomarkers are Acute Phase Proteins (APPs), commonly employed as clinical biomarkers of inflammation in serum but also found in the milk. In particular, the milk isoforms of serum amyloid A (M-SAA) and haptoglobin (HP) (Hussein et al. 2018; Chakraborty et al. 2019; Iliev and Georgieva 2019;) are among the most employed ones. Other proteins indicated as suitable mastitis markers are lactoferrin (LF) (Shimazaki and Kawai 2017) and cathelicidins (CATH) (Smolenski et al. 2011).

Biomarker discovery and implementation are constantly evolving, and comparative data on their diagnostic performances are lacking. Therefore, it is not easy to establish their relative advantages in the different dairy ruminant species compared to the current diagnostic approaches. To provide an organic overview of the topic, to understand if the data currently available in the literature are amenable to meta-analysis, and to attempt a comparative assessment of the respective diagnostic performances, we carried out a literature survey using the systematic review approach based on the PRISMA (Preferred Reporting Items for Systematic Reviews and Meta-Analyses) guidelines. In veterinary medicine, the methodology for systematic reviews has been defined by Sargeant and O'Connor (2020), who identified specific steps to follow. Accordingly, our review question falls within the fourth type, "Diagnostic test accuracy questions", aimed at summarizing diagnostic test accuracy. Specifically, this systematic review aims at examining the scientific literature to answer the diagnostic question: "Which are the diagnostic performances of mastitis protein biomarkers investigated by immunoassays in ruminant milk?".

\section{Methods}

\section{Information sources and search strategy}

We carried out this systematic review according to the guidelines of the PRISMA statement (Moher et al. 2009). We searched three different databases (i.e., MedLine, Scopus, and Web of Science) until January 28, 2021. For Scopus searches, we applied the default search settings (Article title, abstract, and keywords), whereas in Web of Science we used the specific database "Web of Science Core Collection". Our review question falls within the fourth type, "Diagnostic test accuracy questions", aimed at summarizing diagnostic test accuracy, and at answering the diagnostic question: "Which are the diagnostic performances of mastitis protein biomarkers investigated by immunoassays in ruminant milk?" as suggested by Sargeant and O'Connor (2020) for systematic reviews in veterinary medicine. Accordingly, the search terms included the words "biomarker", "marker", "intramammary infection", "mastitis", "milk". These search terms were enriched with the most common markers and detection assays to improve the retrieval of relevant scientific 
articles. Concerning markers, an initial survey of the literature indicated that the ones most associated with the words "milk" and "mastitis" were M-SAA, HP, LF, and CATH. On the other hand, the two immunoassays most frequently used for measuring protein markers devoid of intrinsic enzymatic activity were ELISA and lateral flow/ immunochromatography. Once defined, we combined the search terms and their related Mesh terms into 42 specific searches, as follows: ("biomarker" OR "marker" OR "amyloid" OR "haptoglobin" OR "cathelicidin" OR "lactoferrin") AND ("intramammary infection" OR "mastitis") AND ("milk") AND ("immunoassay" OR "ELISA" OR "lateral flow" OR "immunochromatography") (Supplementary Table I).

\section{Study selection, data extraction, and synthesis method}

Three researchers (AG, ST, and MP) independently screened title, abstract, and full-text for assessing the article compliance with the review question and solved any disagreement by discussion and consensus. When necessary, a fourth researcher with expertise in the field (MFA) was consulted to reach an exclusion decision. Adding to the articles not relating to the review question, we excluded those written in languages different from English and belonging to the categories review, case report, report, book chapter, editorial, abstract, and letter. From each eligible document, the following data were extracted: species, first author, year, country, study design, biomarker, technique, sample type and size, SCC, pathogens, unit of measurement, results, sensitivity, specificity, and cut-off. To synthesize the results we applied the "Synthesis Without Meta-analysis" (SWiM) guidelines (Campbell et al. 2020) by using tables and graphs.

\section{Quality assessment}

The tool consists of 14 questions and two main sections, bias assessment and applicability, including four key domains and three key domains, respectively. In bias assessment, for every study, were assessed the "animal selection" strategy, the "index test", the "reference standard", and "flow and timing". The term "index test" is referred to the test object of study, while "reference standard" to the standard test considered the best available test to diagnose the disease of interest (i.e. a single test, follow-up or combination of tests).

In the applicability assessment, we collected and rated how much the studies matched the review question. For both sections, the risk was expressed as "high", "low", and "unclear" risk when data were insufficient. The 33 screened records showed high heterogeneity in study design, animal selection, and standard reference tests.

\section{Results and discussion}

\section{Results of the PRISMA procedure}

The steps of the literature search are summarized in the PRISMA 2009 flow diagram (Fig. 1). The search led to the identification of 507 scientific papers (220 MedLine +131 Scopus $+156 \mathrm{Web}$ of Science); 16 further records were then added to the original search through an expert revision of the literature, resulting in 523 manuscripts (Supplementary Tables II, III, IV, and V). After removing duplicates, 133 records entered three main screening steps. Firstly, records were screened on the title, secondly on the abstract $(\mathrm{n}=$ 72, intermediate step not included in Fig.1), and finally on the full-text for evaluating the eligibility to qualitative and quantitative analysis (Supplementary Tables VI and VII). As a result of this procedure, 33 scientific articles were considered eligible (Supplementary Table VIII).

\section{Species overview}

By sorting the number of papers based on the dairy species, out of 33 manuscripts, $26(78.8 \%)$ investigated cows, $4(12.1 \%)$ sheep, $3(9.1 \%)$ goats, and $2(6.1 \%)$ buffaloes (Table 1). The total number of records does not match because 2 papers addressed more than one species.

Cow. Out of 26 papers on cow milk, 15 (57.7\%) investigated M-SAA, 9 (34.6\%) HP, 5 (19.2\%) LF, 2 CATH (7.7\%), interleukin $1 \beta$ (IL1 $\beta$ ) and interleukin 6 (IL-6). Other biomarkers were Alpha-1-Acid Glycoprotein (AGP), bovine serum albumin (BSA), C-reactive protein (CRP), immunoglobulin G (IG), interleukin 8 (IL8), interleukin 10 (IL10), interleukin 12 (IL12) lipopolysaccharide-binding protein (LBP), Transforming Growth Factor $\alpha$ (TGF $\alpha$ ), Transforming Growth Factor $\beta$ (TGF $\beta$ ), and Tumor Necrosis Factor $\alpha(\mathrm{TNF} \alpha)$, and they were addressed in 1 paper each (3.8\%) (Table 1). The samples were represented by quarter milk in $18 / 26(69.2 \%)$, and by composite milk in $8 / 26(30.8 \%)$. In one record (Sobczuk-Szul et al. 2014), the milk sample type was not specified, whereas in another study (Thomas et al. 2015) both quarter and composite samples were used. Concerning the diagnostic methods, ELISA was used in 25 (96.2\%) records, whereas in 1 paper $(3.8 \%)$ the biomarker was investigated by SPARCL. Moreover, we observed 25 (96.2\%) observational studies, related to natural inflammation/infection, and only one experimental infection study. Tables 2, 3, 4 and 5 summarize the main findings of the 26 papers evaluating cows. 


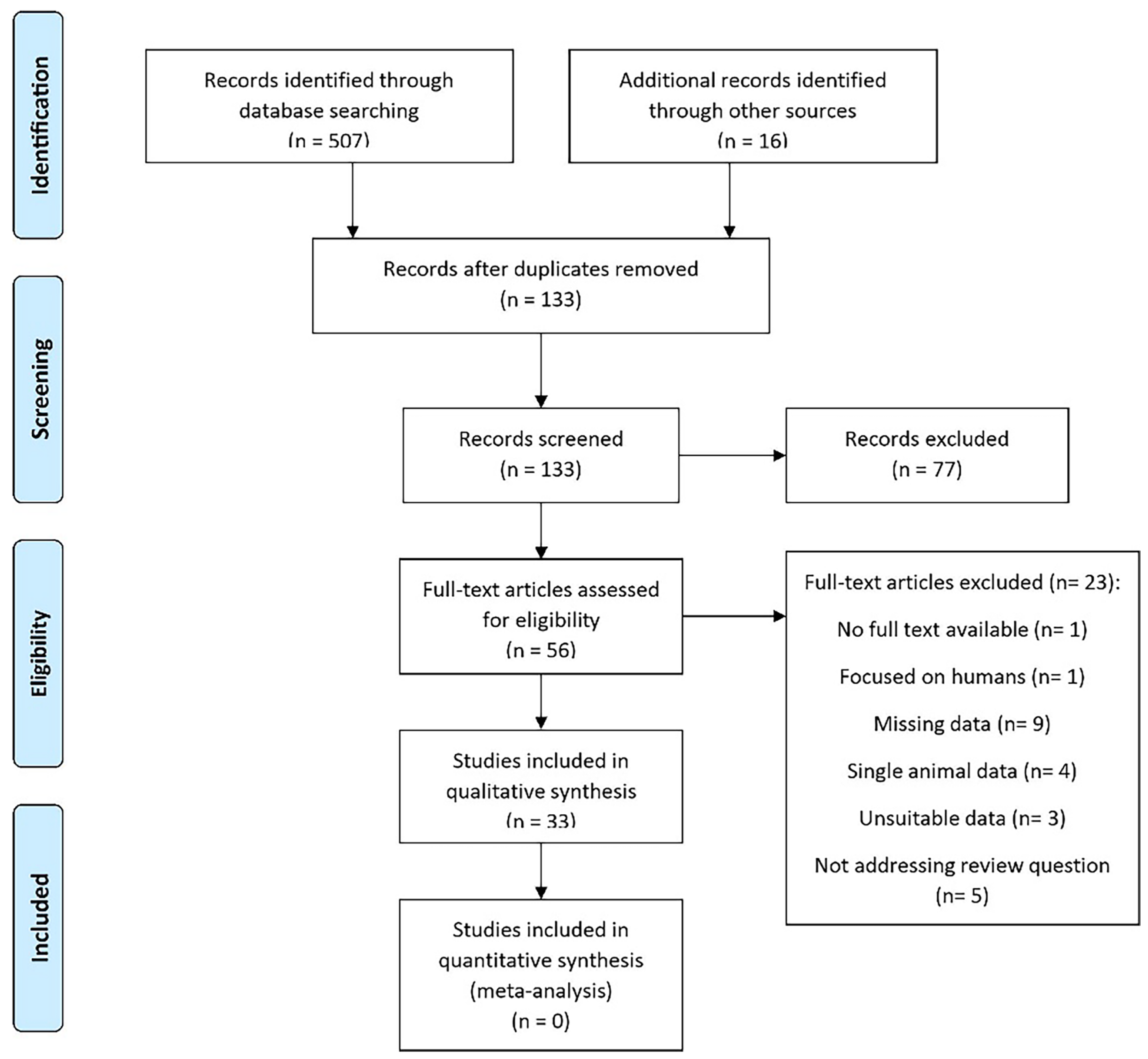

Fig. 1 PRISMA 2009 flow diagram

Sheep. Out of 4 papers on sheep milk, 2 (40.0\%) assessed CATH, while 1 each $(20.0 \%)$ were on interleukins and M-SAA, respectively. ELISA was used in all studies, three of which were observational $(60.0 \%)$ and $2(40.0 \%)$ experimental. All studies were carried out on half-udder milk samples. Table 6 summarizes the main findings of the 4 papers.

Goat Two (66.7\%) out of 3 studies assessed CATH, while 1 (33.3\%) assessed LF. ELISA was used in all studies, which are all observational. All papers investigated biomarkers from half-udder, but one (Chen et al. 2004) used also bulk milk samples. Table 7 summarizes the main findings of the three papers.
Water buffalo Only two observational studies were performed on buffalo. The biomarkers investigated were LF and CATH from quarter milk by ELISA. Table 8 summarizes the main findings of the two papers.

\section{Biomarker overview}

Table 1 summarizes our results presented in descending order of records addressing biomarkers and dairy species. Among all markers, M-SAA was the most frequently mentioned (n. 16; 48.5\%), followed by HP (n. 9; 27.3\%;), CATH (n. 8; 24.2\%) and LF (n. 7; 21.2\%;). Other markers investigated were IL1 $\beta$ and IL6, addressed in 3 papers each $(9.1 \%)$, 
Table 1 Species and biomarker overview

\begin{tabular}{|c|c|c|c|c|c|c|}
\hline Biomarker & $\%$ & $\mathrm{~N}^{\circ}$ & Cow & Sheep & Buffalo & Goat \\
\hline M-SAA & 48.5 & $16 / 33$ & 15 & 1 & - & - \\
\hline HP & 27.3 & $9 / 33$ & 9 & - & - & - \\
\hline СATH & 24.2 & $8 / 33$ & 2 & 2 & 1 & 3 \\
\hline LF & 21.2 & $7 / 33$ & 5 & - & 1 & 1 \\
\hline IL1 $\beta$ & 9.1 & $3 / 33$ & 2 & 1 & - & - \\
\hline IL6 & 9.1 & $3 / 33$ & 2 & 1 & - & - \\
\hline AGP & 3 & $1 / 33$ & 1 & - & - & - \\
\hline BSA & 3 & $1 / 33$ & 1 & - & - & - \\
\hline CRP & 3 & $1 / 33$ & 1 & - & - & - \\
\hline $\mathrm{IgG}$ & 3 & $1 / 33$ & 1 & - & - & - \\
\hline IL8 & 3 & $1 / 33$ & - & 1 & - & - \\
\hline IL10 & 3 & $1 / 33$ & 1 & - & - & - \\
\hline IL12 & 3 & $1 / 33$ & 1 & - & - & - \\
\hline LBP & 3 & $1 / 33$ & 1 & - & - & - \\
\hline TGF $\alpha$ & 3 & $1 / 33$ & 1 & - & - & - \\
\hline TGF $\beta$ & 3 & $1 / 33$ & 1 & - & - & - \\
\hline $\mathrm{TNF} \alpha$ & 3 & $1 / 33$ & 1 & - & - & - \\
\hline
\end{tabular}

Acronyms: M-SAA, milk amyloid A. HP, haptoglobin. CATH, cathelicidins. LF, lactoferrin. IL1 $\beta$, interleukin 1 $\beta$. IL6, interleukin 6. AGP, Alpha-1-Acid Glycoprotein. BSA, bovine serum albumin. CRP, C-reactive protein. IgGs, immunoglobulin G. IL8, interleukin 8. IL10, interleukin 10. IL12, interleukin 12. LBP, lipopolysaccharide-binding protein. TGF $\alpha$, Transforming Growth Factor $\alpha$. TGF $\beta$, Transforming Growth Factor $\beta$. TNF $\alpha$, Tumor Necrosis Factor $\alpha . \%$, percent of articles relating to the numbers of papers addressing the biomarker out of the total. $\mathrm{N}^{\circ}$, number of articles relating to specific biomarker out of the 33 eligible records followed by $\operatorname{IgG}$ (n. $2 ; 6.1 \%$ ) and finally AGP, BSA, CRP, IL8, IL10, IL12, LBP, TGF $\alpha$, TGF $\beta$, TNF $\alpha$ (n. $1 ; 3.0 \%$ ).

Milk serum amyloid (M-SAA) M-SAA is produced extrahepatically by healthy mammary epithelial cells (McDonald et al. 2001; Larson et al. 2005) and during inflammation (Grönlund et al. 2003; Larson et al. 2005; Brenaut et al. 2014). M-SAA was the protein most investigated as subclinical mastitis marker in ruminant milk, particularly in dairy cows (Table 2). In our study, we observed that in 17 papers M-SAA was investigated predominantly by ELISA with the commercial kit Tridelta solid sandwich ELISA in two variants (Tridelta Mast ID range MAA assay, Tridelta Development Ltd., Kildare, Ireland, Cat. No.: TP-802 for serum and TP-807 for milk). However, to diagnose mastitis, the authors did not discriminate for serum or milk amyloid isoforms but for the different matrices, defining the protein as SAA when analyzing serum and M-SAA when analyzing milk, respectively. Interestingly, in 5 studies M-SAA was investigated only by TP-802 (Grönlund et al. 2005; Eckersall et al. 2006b; Kováč et al. 2007; Åkerstedt et al. 2007, 2009), in 5 only by TP-807 (Åkerstedt et al. 2011; Shirazi-Beheshtiha et al. 2011; Jaeger et al. 2017; Hussein et al. 2018; Bochniarz et al. 2020; Wollowski et al. 2021), in 2 by both TP-802 and TP-807 (Gerardi et al. 2009; Safi et al. 2009) and in 5 a Tridelta kit was used but the test category was unspecified
(Suojala et al. 2008; Pyörälä et al. 2011; Kovačević-Filipović et al. 2012; Szczubiał et al. 2012; Thomas et al. 2015). In particular, Gerardi et al. (2009) investigated M-SAA in milk with both TP-807 and TP-802 assays to compare their diagnostic performances. The sensitivity of TP-807 test is 0.10 $\mu \mathrm{g} / \mathrm{ml}$ but a cut-off able to discriminate healthy from mastitic milk has not been defined yet. Miglio et al. (2013) reported a M-SAA peak almost 10 times higher in sheep milk than cow milk. Although no official reference range is fixed for M-SAA in milk, healthy sheep milk concentration ranges from 23.75 to $35.61 \mu \mathrm{g} / \mathrm{ml}$ (Miglio et al. 2013), higher than that observed in cow milk (range: $0.0-7.5 \mu \mathrm{g} / \mathrm{ml}$ ) (Gerardi et al. 2009). In goat, the MAA as mastitis marker was not suitable. In this species, M-SAA levels increase physiologically as lactation progresses as does SCC, even in absence of infection (Pisanu et al. 2020).

Haptoglobin (HP) HP was the second most represented marker in our literature search. Its performance for mastitis detection was analyzed in 9 records, only for cows and by ELISA (Table 3). HP found in milk has an undefined origin. However, similarly to M-SAA, extrahepatic production may also occur in the mammary tissue. Still, it has been demonstrated that HP concentration increases in milk upon endotoxin challenge, experimental, and natural intramammary infection (IMI) (Grönlund et al. 2003; Eckersall et al. 2006; 
Table 2 Cow, results obtained for M-SAA by applying ELISA and SPARCL* (Dalanezi et al. 2020). The unit of measurement is $\mu \mathrm{g} / \mathrm{mL}$.

\begin{tabular}{|c|c|c|c|c|c|c|c|c|c|}
\hline Study & $\begin{array}{l}\text { SCC }(\text { cells } / \mathrm{ml} \mathrm{x} \\
\left.10^{3}\right)\end{array}$ & Pathogens & Sample & Sample group & $\mathrm{N}$ & Value & $\% \mathrm{Se}$ & $\% \mathrm{Sp}$ & $\begin{array}{l}\text { cut- } \\
\text { off }\end{array}$ \\
\hline \multirow[t]{4}{*}{ Kováč et al. 2007} & & & Composite & & & Median / Range & & & \\
\hline & $<100$ & & & SCC group 1 & 5 & $0.67 / 0-1.79$ & & & \\
\hline & $100-400$ & & & SCC group 2 & 5 & $1.52 / 0-7.15$ & & & \\
\hline & $>400$ & & & SCC group 3 & 8 & $26.54 / 1.81-54.28$ & & & \\
\hline \multirow[t]{2}{*}{ Suojala et al. 2008} & & & Quarter & & 7 & $\operatorname{Mean}(S D)$ & & & \\
\hline & & E. coli & & Clinical & & $1.32(0.95)$ & & & \\
\hline \multirow{2}{*}{$\begin{array}{l}\text { Åkerstedt et al. } \\
2009\end{array}$} & Mean $(S D)$ & & Composite & & 68 & Mean $(S D)$ & & & \\
\hline & 218 (179) & & & & & $1.12(1.16)$ & & & \\
\hline \multirow[t]{7}{*}{ Gerardi et al. 2009} & Mean / Median & & Quarter & & & Mean / Median & & & \\
\hline & $618 / 163$ & & & $\begin{array}{l}\text { Subclinical (TP- } \\
\text { 802) }\end{array}$ & 40 & $9.8 / 2.3$ & & & \\
\hline & & & & $\begin{array}{l}\text { Subclinical (TP- } \\
807 \text { ) }\end{array}$ & & $5.5 / 0.5$ & & & \\
\hline & $2,704 / 1,120$ & & & Clinical (TP-802) & 24 & $16.1 / 8.0$ & & & \\
\hline & & & & Clinical (TP-807) & & $6.9 / 3.8$ & & & \\
\hline & $58 / 28$ & & & Healthy (TP-802) & 4 & $0.5 / 0.4$ & & & \\
\hline & & & & Healthy (TP-807) & & $0.1 / 0.1$ & & & \\
\hline \multirow[t]{4}{*}{ Safi et al. 2009} & $\begin{array}{l}\text { Mean }(S D) / \\
\text { Median / Range }\end{array}$ & & Quarter & & & $\begin{array}{l}\text { Mean }(S D) / \\
\text { Median / Range }\end{array}$ & & & \\
\hline & $\begin{array}{l}5,000(9,500) \\
\quad / 1,250 / 3-51,840\end{array}$ & S. aureus & & & 106 & $\begin{array}{l}67(120) / 28 / \\
14-843\end{array}$ & & & \\
\hline & $93(68) / 75 / 3-266$ & Negative & & Healthy & 134 & $9(5) / 8 / 1-29$ & & & \\
\hline & & & & & & & 90.6 & 98.3 & 16.4 \\
\hline \multirow[t]{10}{*}{ Pyörälä et al. 2011} & & & Quarter & & & Median $(I Q R)$ & & & \\
\hline & & & & Subclinical & 136 & $13.4(3-83.5)$ & & & \\
\hline & & & & Clinical & 98 & $22.7(4.4-102.5)$ & & & \\
\hline & & S. aureus & & & 44 & $16.4(3.5-80.2)$ & & & \\
\hline & & NAS & & & 45 & $4.4(1.6-70.5)$ & & & \\
\hline & & S. uberis & & & 43 & $21.2(5.2-99.5)$ & & & \\
\hline & & S. dysgalactiae & & & 48 & $23.9(7.7-85.3)$ & & & \\
\hline & & E. coli & & & 23 & 279.5 (19.7-675.0) & & & \\
\hline & & A. pyogenes & & & 24 & $3.0(<0.3-41.8)$ & & & \\
\hline & & Other & & & 7 & $41.3(3.9-720.0)$ & & & \\
\hline \multirow{3}{*}{$\begin{array}{l}\text { Kovačević-Filipović } \\
\text { et al. } 2012\end{array}$} & Mean (SD) /Range & & Quarter & & & Mean (SD) /Range & & & \\
\hline & $20(2) / 7-31$ & Negative & & Control group & 10 & $1.4(1.1) / 0-4.15$ & & & \\
\hline & $\begin{array}{l}2,066(28) / 556- \\
3,617\end{array}$ & S. aureus & & Subclinical & 10 & $\begin{array}{l}102.6(88.8) / 27.9- \\
324.5\end{array}$ & & & \\
\hline \multirow[t]{4}{*}{$\begin{array}{l}\text { Shirazi-Beheshtiha } \\
\text { et al. } 2011\end{array}$} & & & Quarter & & & $\begin{array}{l}\text { Mean }(S D) / \\
\text { Median / Range }\end{array}$ & & & \\
\hline & $<130$ & Negative & & Healthy & 38 & $\begin{array}{l}0.61(0.5) / 0.44 / \\
0.1-1.9\end{array}$ & & & \\
\hline & $>130$ & Positive & & Subclinical & 52 & $\begin{array}{l}12.83(12.8) / 7.88 / \\
0.84-50.5\end{array}$ & & & \\
\hline & & & & & & & 92.3 & 92.1 & 1.6 \\
\hline $\begin{array}{l}\text { Szczubiał et al. } \\
2012\end{array}$ & & & Quarter & & & Mean (SD) /Range & & & \\
\hline
\end{tabular}


Table 2 (continued)

\begin{tabular}{|c|c|c|c|c|c|c|c|c|c|}
\hline Study & $\begin{array}{l}\text { SCC }(\text { cells } / \mathrm{ml} \mathrm{x} \\
\left.10^{3}\right)\end{array}$ & Pathogens & Sample & Sample group & $\mathrm{N}$ & Value & $\% \mathrm{Se}$ & $\% \mathrm{Sp}$ & $\begin{array}{l}\text { cut- } \\
\text { off }\end{array}$ \\
\hline & & S. aureus & & Subclinical & 12 & $\begin{array}{l}41.75(26.6) / 8.76- \\
74.75\end{array}$ & & & \\
\hline & & S. agalactiae & & & 9 & $\begin{array}{c}60.11(17.53) / \\
9.78-111.26\end{array}$ & & & \\
\hline & & S. dysgalactiae & & & 18 & $\begin{array}{r}72.43(43.22) / \\
19.90-121.20\end{array}$ & & & \\
\hline & & S. uberis & & & 18 & $\begin{array}{c}92.23(28.64) / \\
8.58-221.64\end{array}$ & & & \\
\hline & & NAS & & & 19 & $\begin{array}{l}12.47(6.95) / 6.53- \\
23.33\end{array}$ & & & \\
\hline & & Candida spp. & & & 8 & $\begin{array}{c}101.00(55.56) / \\
9.61-199.36\end{array}$ & & & \\
\hline & & Negative & & Healthy & 14 & $\begin{array}{l}11.67(7.40) / 5.24- \\
19.04\end{array}$ & & & \\
\hline \multirow{11}{*}{$\begin{array}{l}\text { Wollowski et al. } \\
2021\end{array}$} & & & Quarter & & & Mean & & & \\
\hline & & & & Subclinical & 107 & 2.62 & & & \\
\hline & & & & Clinical (all) & 201 & 6.67 & & & \\
\hline & & & & Clinical (mild) & 45 & 6.14 & & & \\
\hline & & & & Clinical (moderate) & 95 & 5.69 & & & \\
\hline & & & & Clinical (severe) & 61 & 8.63 & & & \\
\hline & & & & Healthy & 67 & 1.06 & & & \\
\hline & & S. aureus & & Subclinical & 12 & 4.38 & & & \\
\hline & & NAS & & Subclinical & 14 & 1.57 & & & \\
\hline & & & & & & & 65 & 76 & 1.28 \\
\hline & & & & & & & 77 & 83 & 1.81 \\
\hline \multirow[t]{5}{*}{ Thomas et al. 2015} & Range & & Composite & & & Median / Range & & & \\
\hline & $9-6,154$ & & & All & 54 & $1.17 /<0.6-50.13$ & & & \\
\hline & $<100$ & & & Healthy & 29 & $0.6 /<0.6-50.13$ & & & \\
\hline & $101-200$ & & & Subclinical & 8 & $0.6 /<0.6$ & & & \\
\hline & $>200$ & & & Clinical & 17 & $0.6 /<0.6-24.81$ & & & \\
\hline \multirow[t]{3}{*}{ Hussein et al. 2018} & & & Quarter & & & Mean & & & \\
\hline & $\leq 500$ & & & SCC group 1 & 148 & 3.58 & & & \\
\hline & $\geq 500$ & & & SCC group 2 & 72 & 35.2 & & & \\
\hline \multirow{5}{*}{$\begin{array}{l}\text { Bochniarz et al. } \\
2020\end{array}$} & & & Quarter & & & Median / Range & & & \\
\hline & & S. uberis & & Clinical & 14 & $2.59 / 0.47-5.84$ & & & \\
\hline & & S. agalactiae & & & 7 & $3.88 / 0.88-7.60$ & & & \\
\hline & & Strep. spp. & & & 30 & $1.13 / 0.42-7.60$ & & & \\
\hline & $<100$ & Negative & & Healthy & 10 & $0.32 / 0.15-0.51$ & & & \\
\hline Jaeger et al. 2017 & Median $(I Q R)$ & & Composite & & & Median $(I Q R)$ & & & \\
\hline
\end{tabular}


Table 2 (continued)

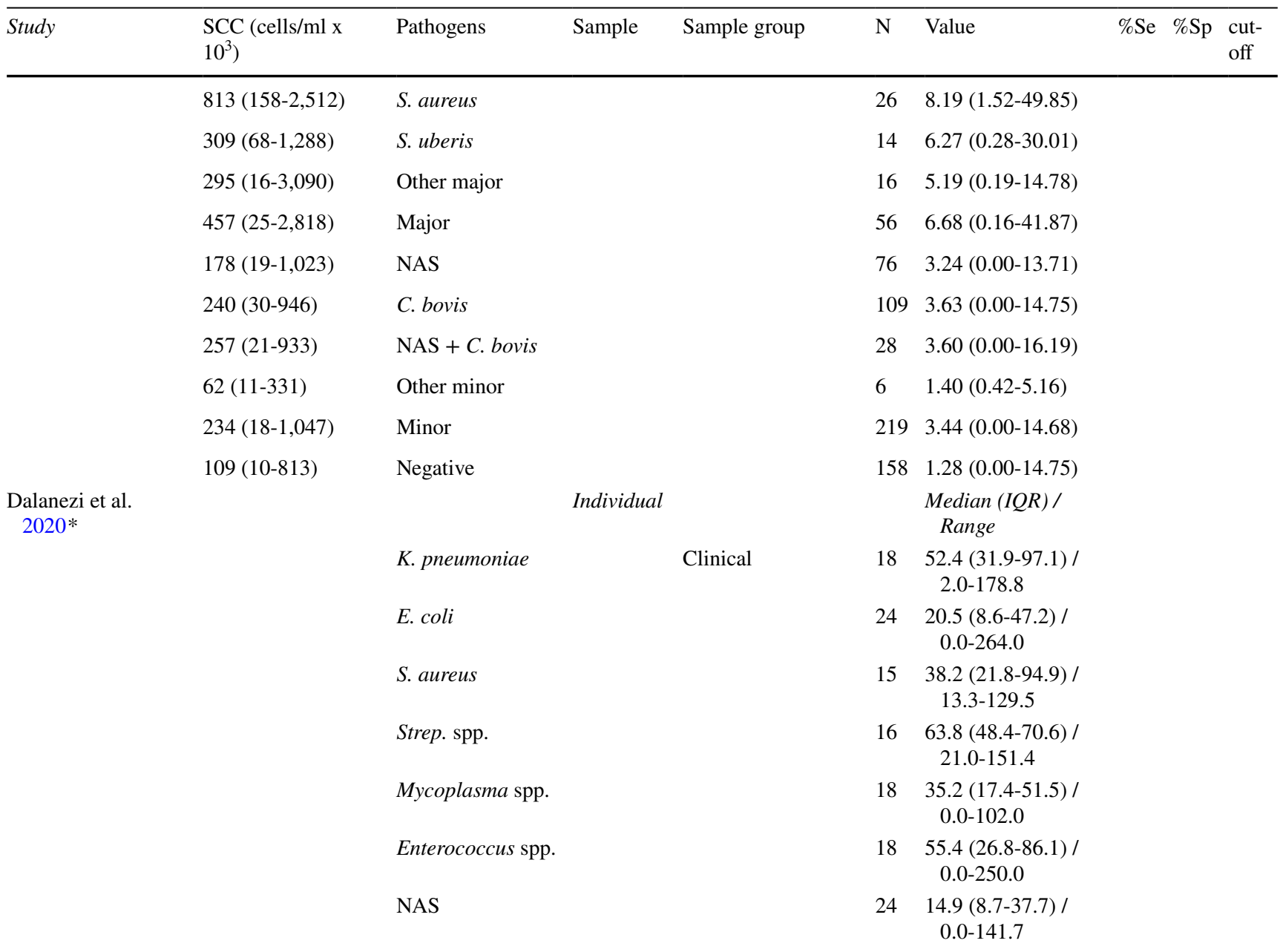

Gerardi et al. 2009). Interestingly, HP appears in milk and raises in level 3 hours and in blood 9 hours after inflammation (Hiss et al. 2004), indicating that the production of this biomarker by the mammary gland is rapid and specific. The diagnostic performance reported in cows by various authors is promising (Table 3 ) and encourages its evaluation also in other dairy species. For its characteristics, this biomarker might also be promising for the diagnosis of caprine mastitis, particularly in late lactation, when the SCC is high and other markers fail to provide satisfactory performances (Pisanu et al. 2020).

Cathelicidin (CATH) CATH was measured mainly most by ELISA in goats (n. 3), cows (n. 2), sheep (n. 2), and water buffalo (n. 1). CATH are host defense proteins with antimicrobial and immunomodulatory functions (van Harten et al. 2018) produced by milk PMNs (Kościuczuk et al. 2012) and mammary epithelial cells (Zanetti 2004, 2005; Addis et al. 2013; Cubeddu et al. 2017). The ruminant genome contains numerous CATH proteoform genes, but their differential abundance in mastitic milk is poorly known (Zanetti 2005).
CATH showed a high diagnostic performance especially in cows and sheep, also in late lactation. Interestingly, by using a threshold set using negative healthy controls, a good sensitivity of the dedicated ELISA is reached not only for cow and sheep milk (Addis et al. 2016a, 2016b), but also for water buffalo milk (Puggioni et al. 2020a). Conversely, the application of CATH-ELISA in goats remains unsatisfactory in late lactation, especially in pluriparous goats. In fact, the related physiological increase in PMN compromises its reliability, as mentioned above for M-SAA (Pisanu et al. 2020).

Lactoferrin (LF) LF was primarily detected by ELISA in studies involving cows (n. 5), goats (n. 1,) and water buffalo (n. 1). LF is a glycoprotein of the immune defense secreted by mammary epithelial cells during the late stage of milking and mammary involution (Welty et al. 1976; Galfi et al. 2016a). The presence of LF in milk is due to secretion by epithelial cells and degranulation of PMNs during inflammation (Lash et al. 1983). Even though LF is not an APP, it increases remarkably during the inflammatory response due to its production by mammary epithelial cells (Galfi 
Table 3 Cow, results obtained for haptoglobin by ELISA. The unit of measurement is $\mu \mathrm{g} / \mathrm{mL}$.

\begin{tabular}{|c|c|c|c|c|c|c|c|c|c|}
\hline Study & $\begin{array}{l}\mathrm{SCC}(\text { cells } / \mathrm{ml} \\
\left.\mathrm{x} 10^{3}\right)\end{array}$ & Pathogens & Sample & Sample group & $\mathrm{N}$ & Value & $\% \mathrm{Se}$ & $\% \mathrm{Sp}$ & cutoff \\
\hline \multirow[t]{11}{*}{ Hiss et al. 2007} & Median / Range & & Quarter & & & Median / Range & & & \\
\hline & $40 / 4-1,512$ & Negative & & Group 1 & 79 & $0.70 / 0.35-16.0$ & & & \\
\hline & $\begin{array}{l}70,500 / \\
6-2,249\end{array}$ & C. bovis & & Group 2 & 70 & $1.85 / 0.35-85.0$ & & & \\
\hline & $136 / 12-10,000$ & Mixed inf. & & Group 3 & 45 & $2.4 / 0.5-150.2$ & & & \\
\hline & $167 / 9-4,171$ & NAS & & Group 4 & 60 & $3.1 / 0.35-576.0$ & & & \\
\hline & 405 / 32-10,000 & Strep. spp. & & Group 5 & 29 & $4.4 / 0.50-974.0$ & & & \\
\hline & 335 / 8-8,804 & $\begin{array}{l}\text { Mixed }+S . \\
\text { aureus }\end{array}$ & & Group 6 & 35 & $4.8 / 0.35-232.4$ & & & \\
\hline & $\begin{array}{l}1,741 / \\
16-10,000\end{array}$ & S. aureus & & Group 7 & 49 & $\begin{array}{l}39.6 / 0.35- \\
304.8\end{array}$ & & & \\
\hline & $\begin{array}{l}8,861 / 1,658- \\
10,000\end{array}$ & E. coli & & Group 8 & 3 & $\begin{array}{l}81.0 / 59.0- \\
184.0\end{array}$ & & & \\
\hline & & & & & & & 85 & 92 & $2.2(\mathrm{SCC} 100)$ \\
\hline & & & & & & & 89 & 92 & $2.7($ SCC 200$)$ \\
\hline \multirow{4}{*}{$\begin{array}{l}\text { Kováč et al. } \\
2007\end{array}$} & & & Composite & & & Median / Range & & & \\
\hline & $<100$ & & & Group 1 & 7 & $0 / 0-0$ & & & \\
\hline & $101-400$ & & & Group 2 & 7 & $0 / 0-0.68$ & & & \\
\hline & $>400$ & & & Group 3 & 8 & $6.76 / 0-20.0$ & & & \\
\hline \multirow[t]{4}{*}{ Safi et al. 2009} & Median / Range & & Quarter & & & Median / Range & & & \\
\hline & $\begin{array}{l}1,250 / \\
3-51,840\end{array}$ & $\begin{array}{l}\text { S. aureus, } \\
\text { Strep. ag. }\end{array}$ & & Subclinical & 39 & $10 / 0-1,382$ & & & \\
\hline & 75 / 3-266 & Negative & & Healthy & 134 & $0 / 0-500$ & & & \\
\hline & & & & & & & 90.6 & 68.6 & 3.9 \\
\hline \multirow[t]{3}{*}{ Zeng et al. 2009} & & & Quarter & & & Mean (SD) & & & \\
\hline & $<250$ & & & SCC group 1 & 347 & $0.50(0.15)$ & & & \\
\hline & $>250$ & & & SCC group 2 & 46 & $7.18(2.10)$ & & & \\
\hline \multirow{9}{*}{$\begin{array}{l}\text { Wenz et al. } \\
2010\end{array}$} & & & Quarter & & & Mean $(95 \%$ CI $)$ & & & \\
\hline & & & & Clinical (mild) & 87 & $503(344-735)$ & & & \\
\hline & & & & $\begin{array}{l}\text { Clinical (moder- } \\
\text { ate-severe) }\end{array}$ & 60 & $\begin{array}{l}1,013(644- \\
1594)\end{array}$ & & & \\
\hline & & Gram-negative & & & 83 & $\begin{array}{c}1,126(759- \\
1,670)\end{array}$ & & & \\
\hline & & Gram-positive & & & 64 & $575(375-881)$ & & & \\
\hline & & NAS & & & 19 & $403(196-828)$ & & & \\
\hline & & Strep. spp. & & & 45 & $686(418-1,127)$ & & & \\
\hline & & E. coli & & & 57 & $\begin{array}{c}1,052(675- \\
1,639)\end{array}$ & & & \\
\hline & & Coliforms & & & 26 & $\begin{array}{l}1,370(704- \\
2,666)\end{array}$ & & & \\
\hline $\begin{array}{l}\text { Pyörälä et al. } \\
2011\end{array}$ & & & Quarter & & & Median $(I Q R)$ & & & \\
\hline
\end{tabular}


Table 3 (continued)

\begin{tabular}{|c|c|c|c|c|c|c|c|c|c|}
\hline Study & $\begin{array}{l}\mathrm{SCC}(\text { cells } / \mathrm{ml} \\
\left.\times 10^{3}\right)\end{array}$ & Pathogens & Sample & Sample group & $\mathrm{N}$ & Value & $\% \mathrm{Se}$ & $\% \mathrm{Sp}$ & cutoff \\
\hline & & S. aureus & & & 44 & $\begin{array}{l}33.0(<7.8- \\
95.3)\end{array}$ & & & \\
\hline & & NAS & & & 45 & $7.8(<7.8-73)$ & & & \\
\hline & & Strep. uberis & & & 43 & $\begin{array}{l}36.7(<7.8- \\
249.5)\end{array}$ & & & \\
\hline & & E. coli & & & 23 & $\begin{array}{l}243.5(32.1- \\
625)\end{array}$ & & & \\
\hline & & A. pyogenes & & & 24 & $\begin{array}{l}440.3(164.5- \\
961.5)\end{array}$ & & & \\
\hline & & $\begin{array}{l}\text { Strep. dysgalac- } \\
\text { tiae }\end{array}$ & & & 48 & $\begin{array}{l}34.5(<7.8- \\
125.5)\end{array}$ & & & \\
\hline & & Other bacteria & & & 7 & $\begin{array}{c}<7.8(<7.8- \\
159.5)\end{array}$ & & & \\
\hline & & & & Subclinical & 136 & $\begin{array}{c}33.8(<7.8- \\
135.5)\end{array}$ & & & \\
\hline & & & & Clinical & 98 & $80(10.2-332.0)$ & & & \\
\hline & $\begin{array}{l}>200 \\
>100\end{array}$ & & & & & & 52.974 .0 & 94.664 .4 & \\
\hline \multirow{6}{*}{$\begin{array}{l}\text { Thomas et al. } \\
2015\end{array}$} & Median / Range & & & & & Median / Range & & & \\
\hline & & & Quarter & All & 149 & $3.60 /<0.4-420$ & & & \\
\hline & $101-200$ & & Composite & Subclinical & 8 & $4.02 /<0.4-5.28$ & & & \\
\hline & $>200$ & & & Clinical & 17 & $\begin{array}{l}6.40 /<0.4- \\
55.46\end{array}$ & & & \\
\hline & $96 / 9-6,154$ & & & All & 54 & $\begin{array}{l}3.46 /<0.4- \\
55.46\end{array}$ & & & \\
\hline & $<100$ & & & Healthy & 29 & $\begin{array}{l}2.96 /<0.4- \\
13.74\end{array}$ & & & \\
\hline \multirow[t]{8}{*}{$\begin{array}{l}\text { Dalanezi et al. } \\
2020\end{array}$} & & & Individual & & & $\begin{array}{l}\text { Median }(I Q R) / \\
\text { Range }\end{array}$ & & & \\
\hline & & K. pneumoniae & & Clinical & 18 & $\begin{array}{l}206.1(126.3- \\
468.1) / 0.0- \\
1,113.3\end{array}$ & & & \\
\hline & & E. coli & & & 24 & $\begin{array}{l}164.1(71.7- \\
305.1) / 0.0- \\
2,009.4\end{array}$ & & & \\
\hline & & S. aureus & & & 15 & $\begin{array}{l}158.7(0.0- \\
300.0) / 0.0- \\
596.1\end{array}$ & & & \\
\hline & & Strep. spp. & & & 16 & $\begin{array}{l}179.0(130.2- \\
363.7) / 0.0- \\
812.2\end{array}$ & & & \\
\hline & & $\begin{array}{l}\text { Mycoplasma } \\
\text { spp. }\end{array}$ & & & 18 & $\begin{array}{l}102.0(0.0- \\
332.8) / 0.0- \\
582.9\end{array}$ & & & \\
\hline & & $\begin{array}{l}\text { Enterococcus } \\
\text { spp. }\end{array}$ & & & 18 & $\begin{array}{c}43.0(0.0-127.3) \\
\quad / 0.0-213.0\end{array}$ & & & \\
\hline & & NAS & & & 24 & $\begin{array}{c}0.0(0.0-66.3) / \\
0.0-319.1\end{array}$ & & & \\
\hline $\begin{array}{l}\text { Wollowski et al. } \\
2021\end{array}$ & & & Quarter & & & Mean & & & \\
\hline
\end{tabular}


Table 3 (continued)

\begin{tabular}{|c|c|c|c|c|c|c|c|c|c|}
\hline Study & $\begin{array}{l}\mathrm{SCC}(\text { cells } / \mathrm{ml} \\
\left.\mathrm{x} 10^{3}\right)\end{array}$ & Pathogens & Sample & Sample group & $\mathrm{N}$ & Value & $\% \mathrm{Se}$ & $\% \mathrm{Sp}$ & cutoff \\
\hline & & & & Subclinical & 107 & 10.15 & & & \\
\hline & & & & Clinical (all) & 115 & 13.73 & & & \\
\hline & & & & Healthy & 67 & 0.98 & & & \\
\hline & & Strep. uberis & & Subclinical & 22 & 11.1 & & & \\
\hline & & S. aureus & & Subclinical & 12 & 11.86 & & & \\
\hline & & NAS & & Subclinical & 14 & 8.52 & & & \\
\hline & & & & & & & 92 & 94 & 3.65 \\
\hline & & & & & & & 96 & 99 & 5.40 \\
\hline
\end{tabular}

et al. 2016a). Concerning test characteristics for goats and cows, two studies carried out a competitive ELISA by using a lactoferrin antiserum from rabbit, and goat lactoferrin was isolated and purified (Chen and Mao 2004; Chen et al. 2004). In other studies, cow LF was quantified by a commercial sandwich LF ELISA kit (Bethyl Laboratories, Montgomery, TX) (Cheng et al. 2008; Sobczuk-Szul et al. 2014; Galfi et al. 2016a, 2016b). For water buffalo, a specific ELISA kit was produced for the study (Özenç et al. 2019). None of the studies reported test characteristics for LF, and therefore no information on sensitivity or specificity is available for this marker.

Other markers IL1 $\beta$ and IL6 were studied in both cows and sheep (Tab.2), IL-8 only in sheep, and the other proteins (AGP, BSA, CRP, IG, IL10, IL12, LBP, TGF $\alpha$, TGF $\beta$, $\mathrm{TNF} \alpha$ ) only in cows. In humans, immune cytokines such as TNF $\alpha$, INF $\gamma$, and ILs are investigated as inflammatory markers to detect subclinical mastitis and identify Th1/Th2 ratio in the inflammatory process (Tuaillon et al. 2017). CRP was studied as a predictor of severity of symptomatology in women's breast inflammation (Fetherston et al. 2006).

In cows, immune cells and their related cytokines have been the subject of recent studies (Gulbe et al. 2020; Shaheen et al. 2020), especially pro-inflammatory immune mediators. In other dairy ruminants, however, these proteins and their roles in mastitis have to be still studied and understood.

\section{Method overview}

Clinical signs, SCC or CMT, and bacteriological culture results were the reference standard methods used to define the presence of mastitis or IMI in dairy ruminants, in association or alone (Chakraborty et al. 2019). Among the analytical techniques applied to evaluating protein biomarkers, ELISA was used in 31 of 33 (93.9\%) selected records, whereas SPARCL (Spatial Proximity Analyte) and RID (radial immunodiffusion) were each applied in 1 paper.

\section{Limitations of the systematic review}

Issues in research methodology Our research encountered several critical issues in applying the PRISMA standard methodology, especially concerning the search strategy. While selecting the best performing keywords for carrying out our review, we assessed several combinations for finding those enabling to collect the most comprehensive but selective set of publications possible. During the process, we had some unexpected findings; for instance, including the keyword "ruminant" produced a less sensitive search, leading to the decision to remove it. Interestingly, this gives a clue that the word "ruminant" is uncommonly used in title, abstract, or keywords, probably because the authors prefer to report only the name of the dairy species. Furthermore, misleading titles and abstracts led to identifying papers that did not address the research question, and these had to be excluded (as detailed in Methods). On the other hand, we compensated for the possible loss of records consequent to improper index terms with an additional critical revision of the literature performed on PubMed by an expert author. Furthermore, the references of each retrieved article were screened as a further compensative measure. Nevertheless, there is always a risk for exclusion for those articles that do not contain at least one of the selected search terms in the title, abstract, or keywords. Therefore, it is very important that the authors pay particular care when drafting these crucial parts in order to maximize article retrieval.

Bias assessment and applicability of studies Defining quality assessment of primary studies is an essential step in systematic reviews. Therefore, the risk of bias and applicability must be evaluated and scored in all studies, especially those focused on diagnostic accuracy. Hence, we applied QUADAS, a quality assessment tool, to all the selected studies. 


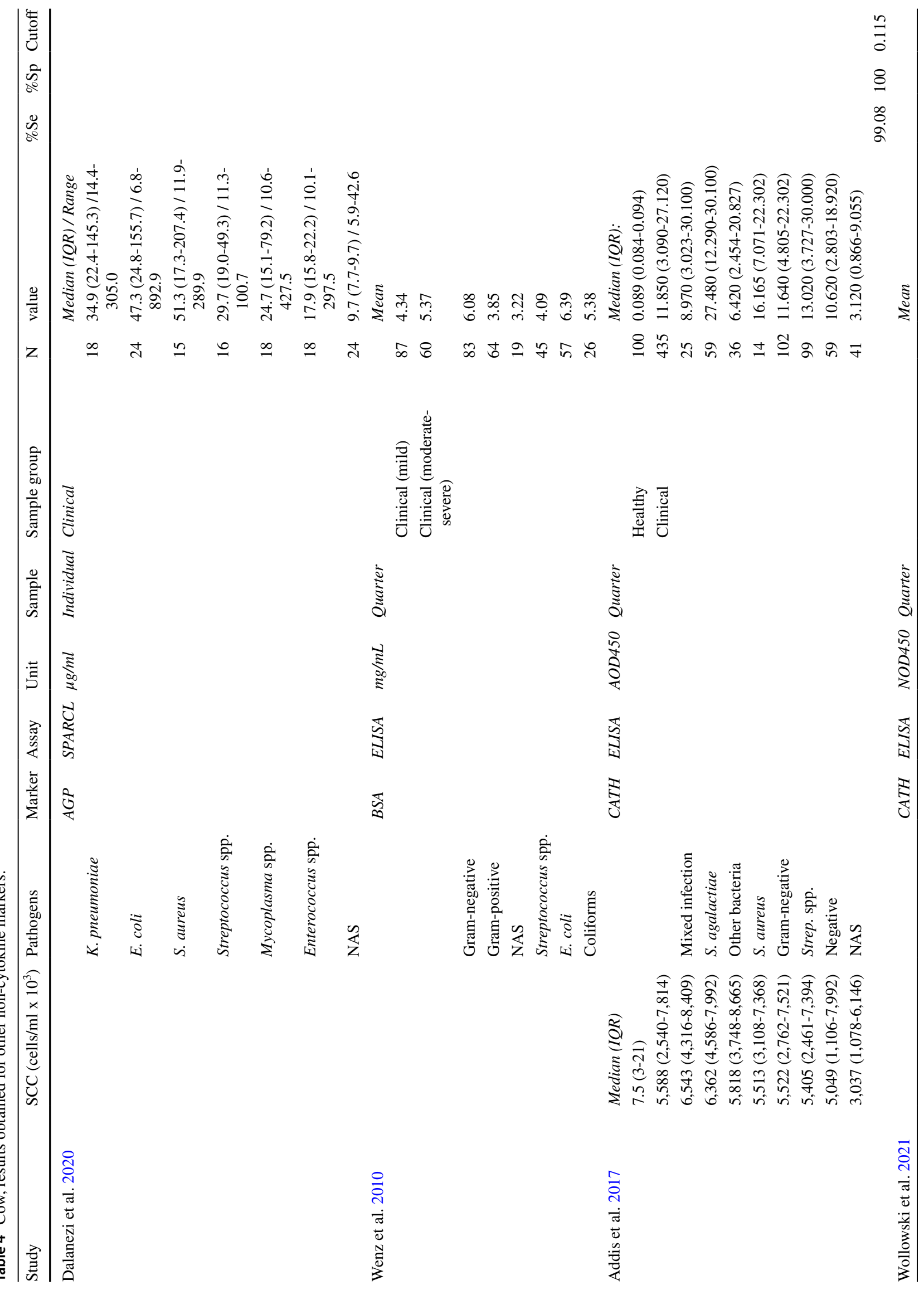




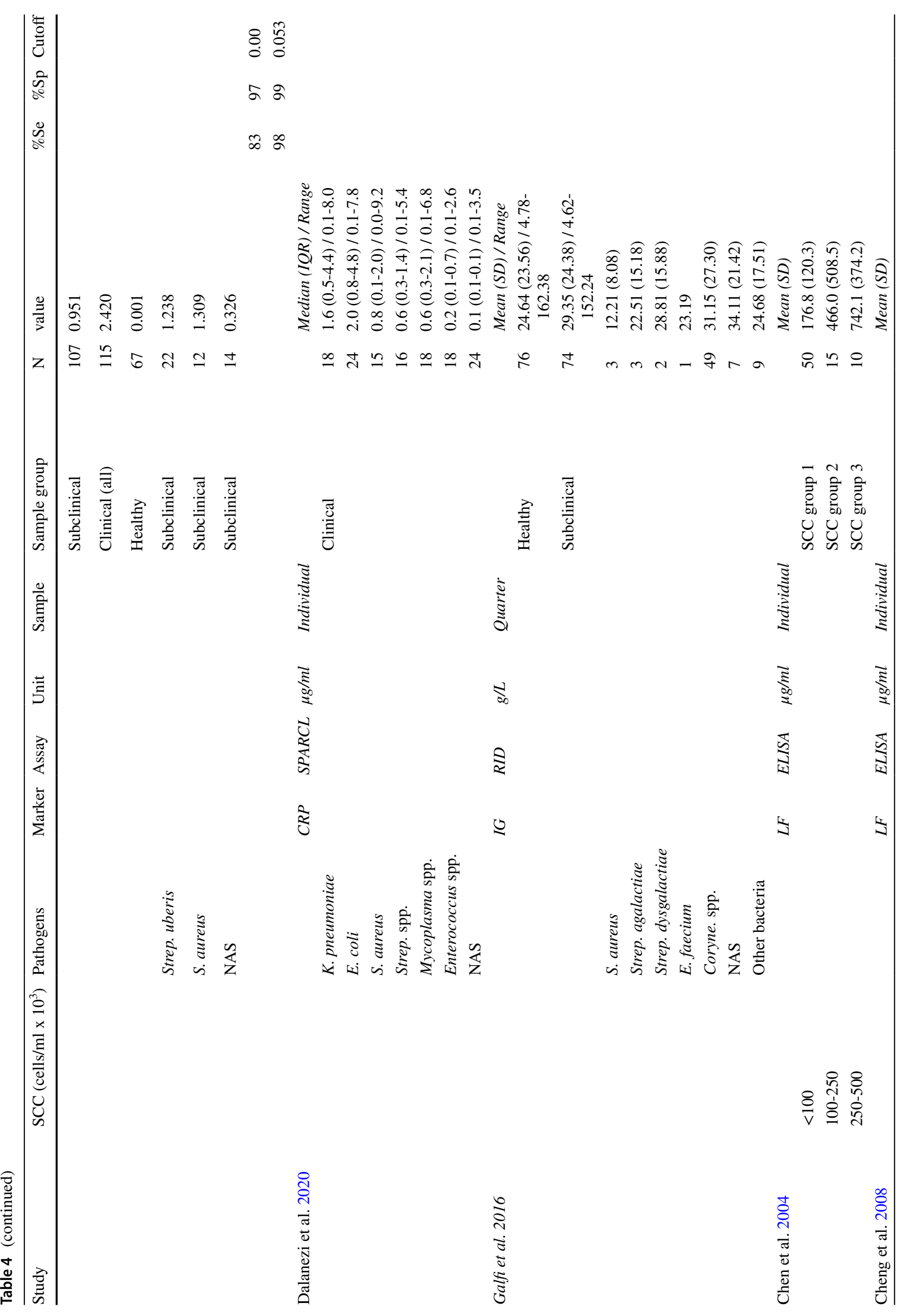




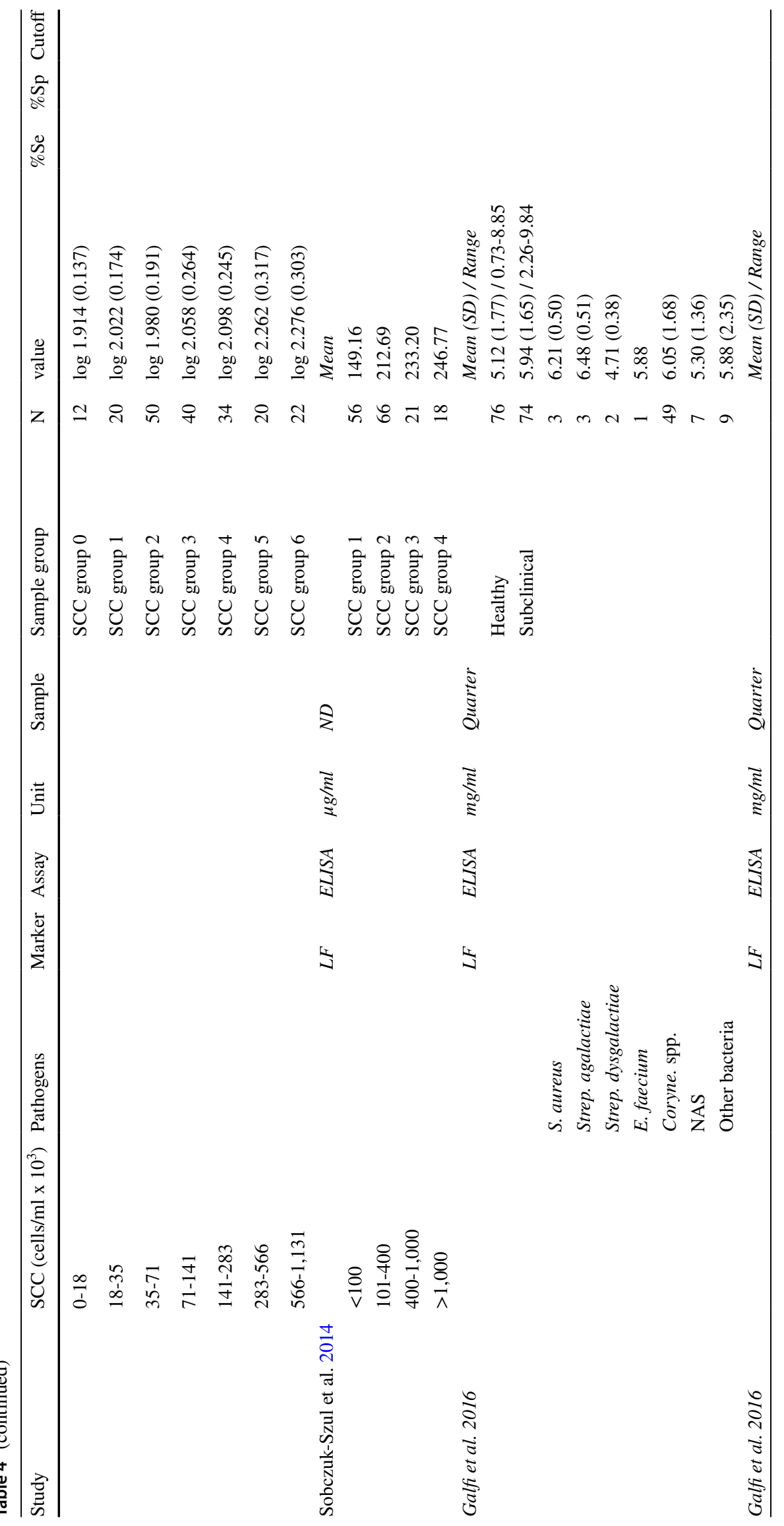




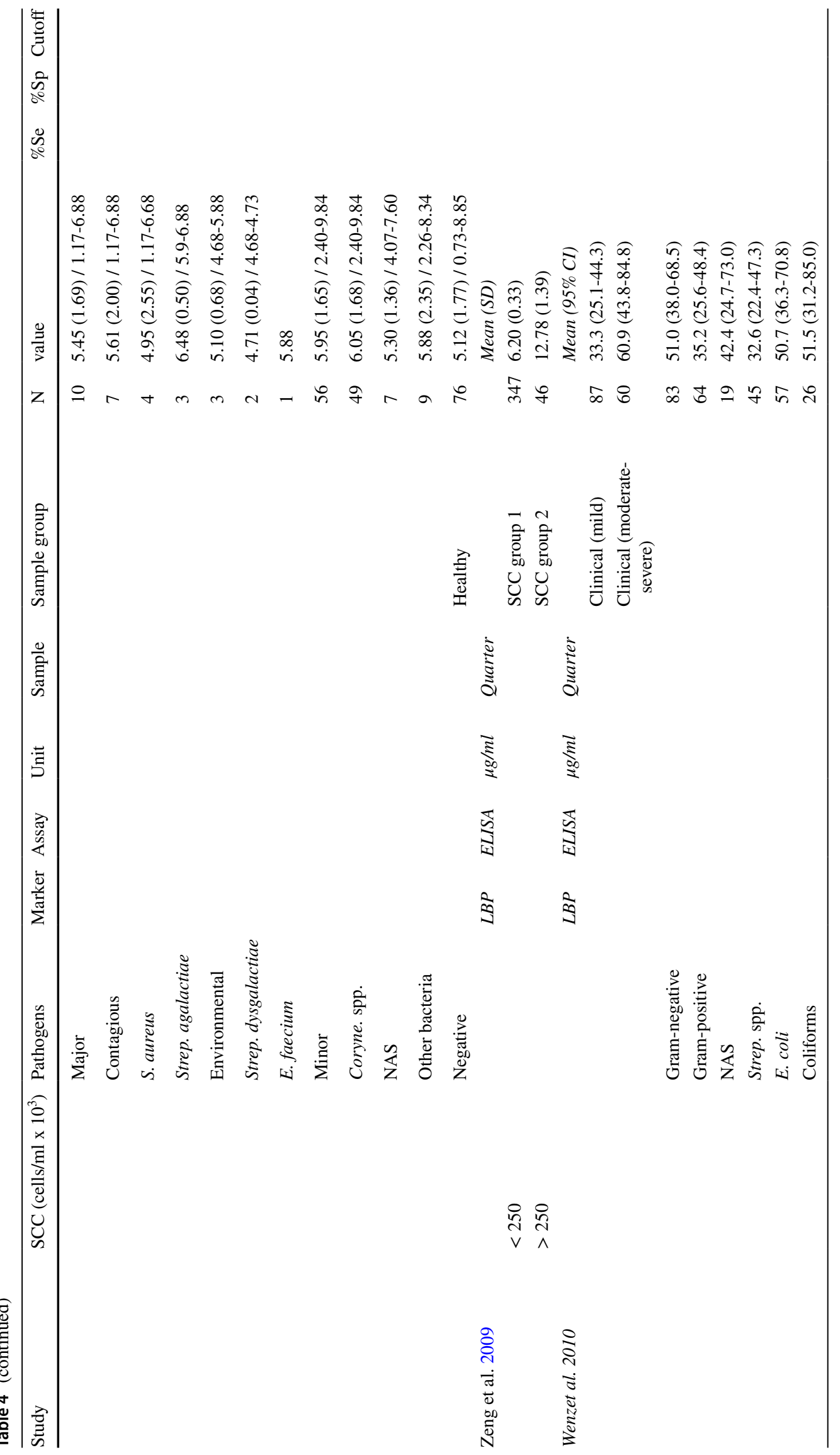




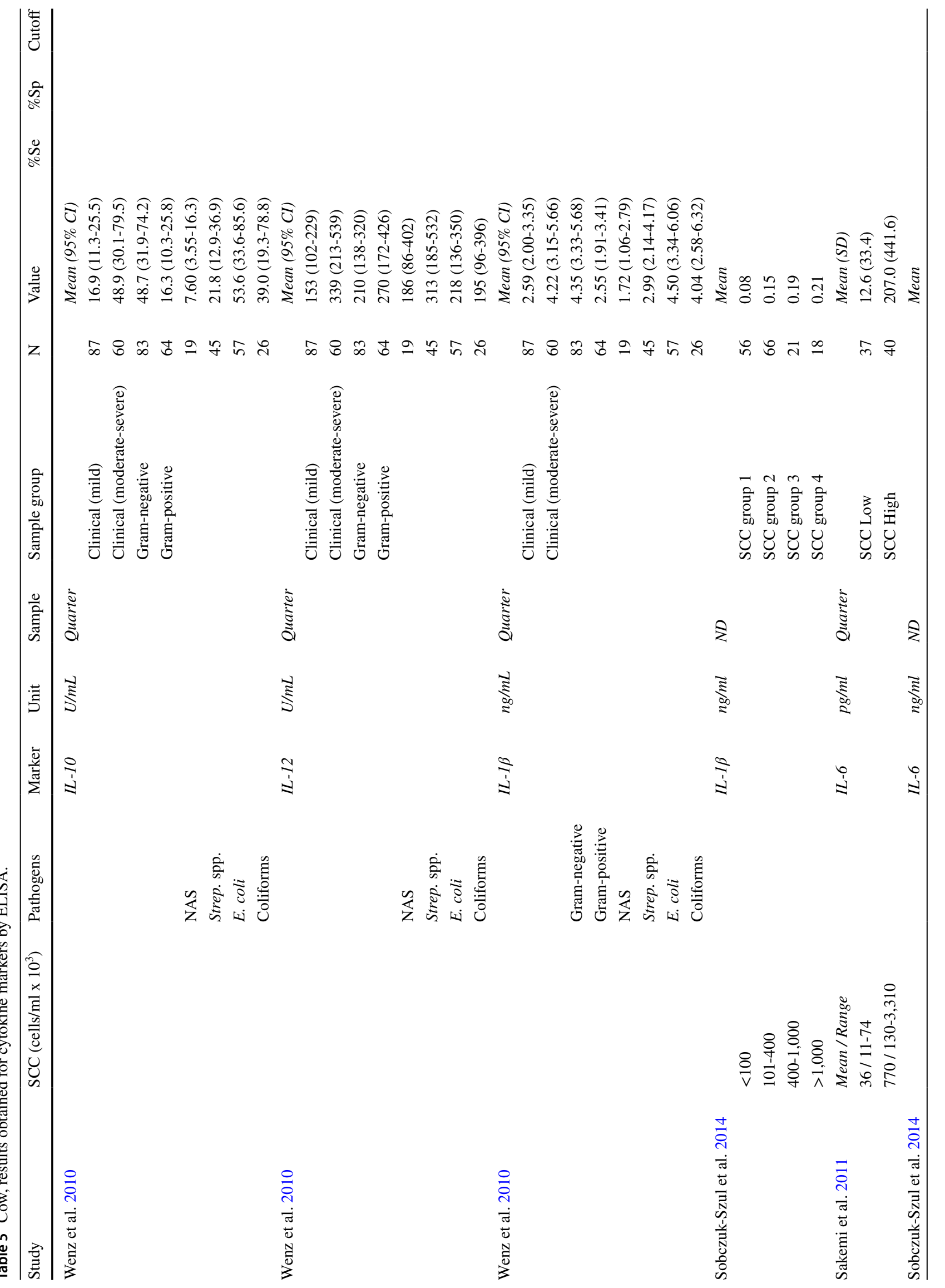




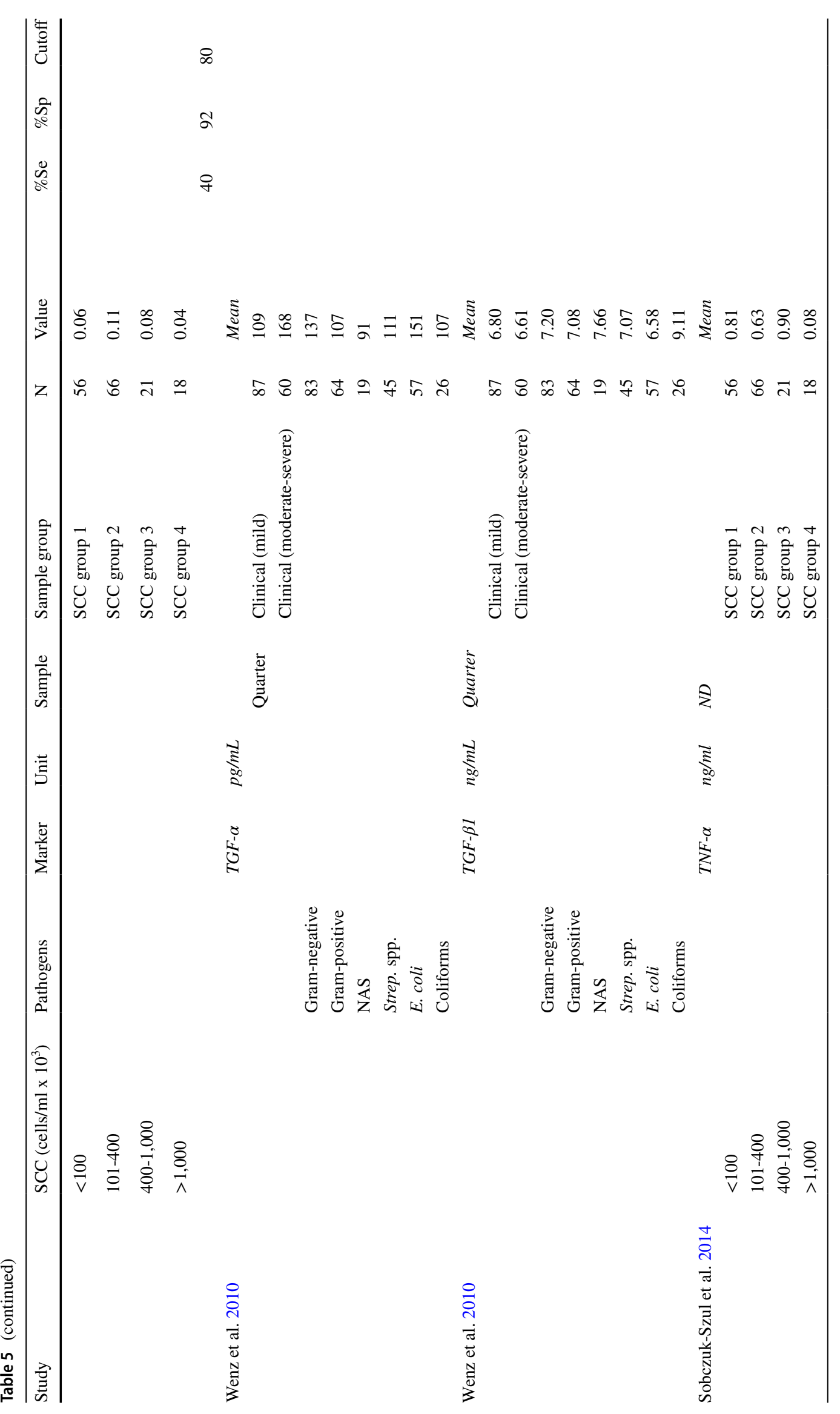




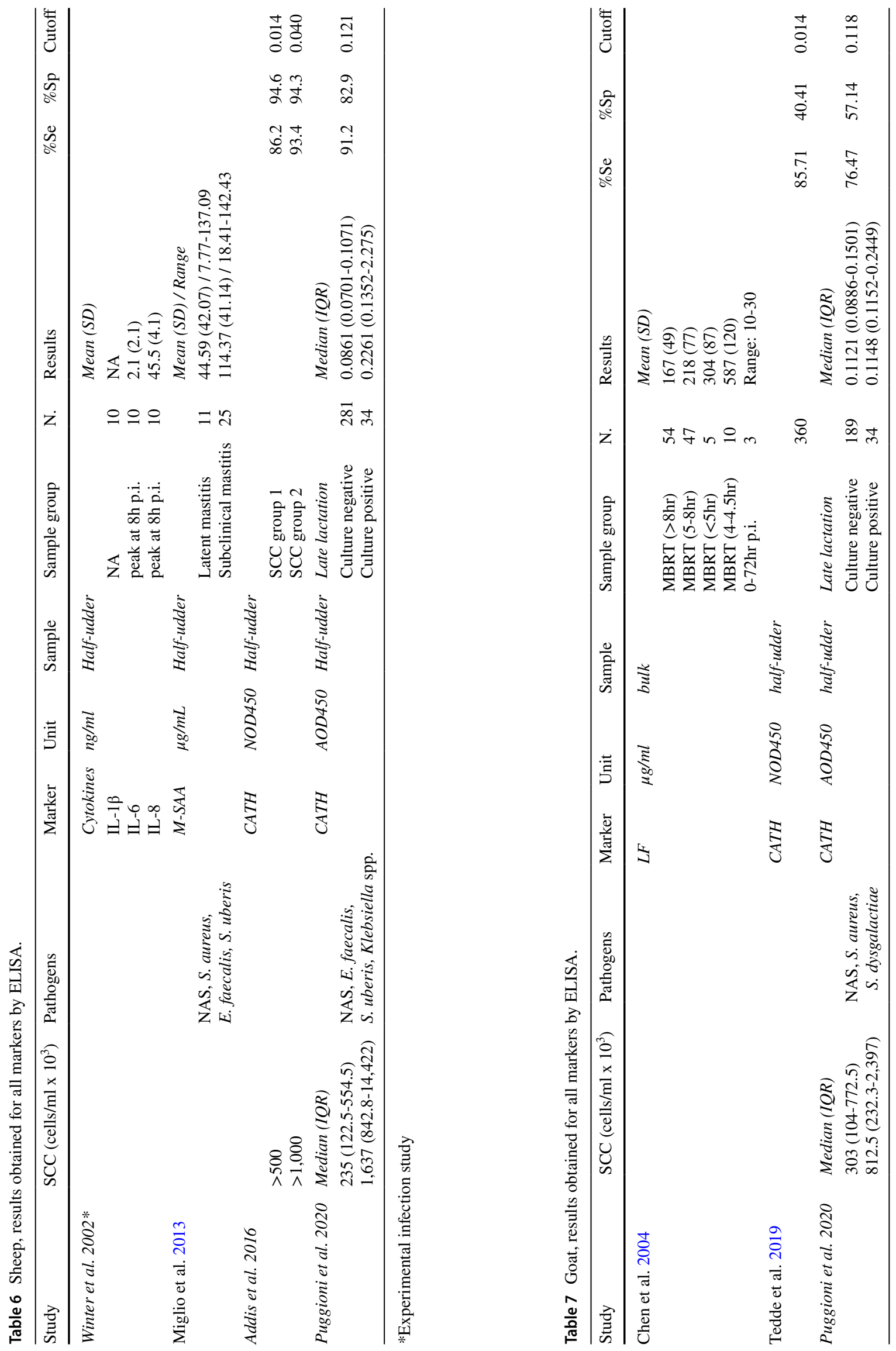




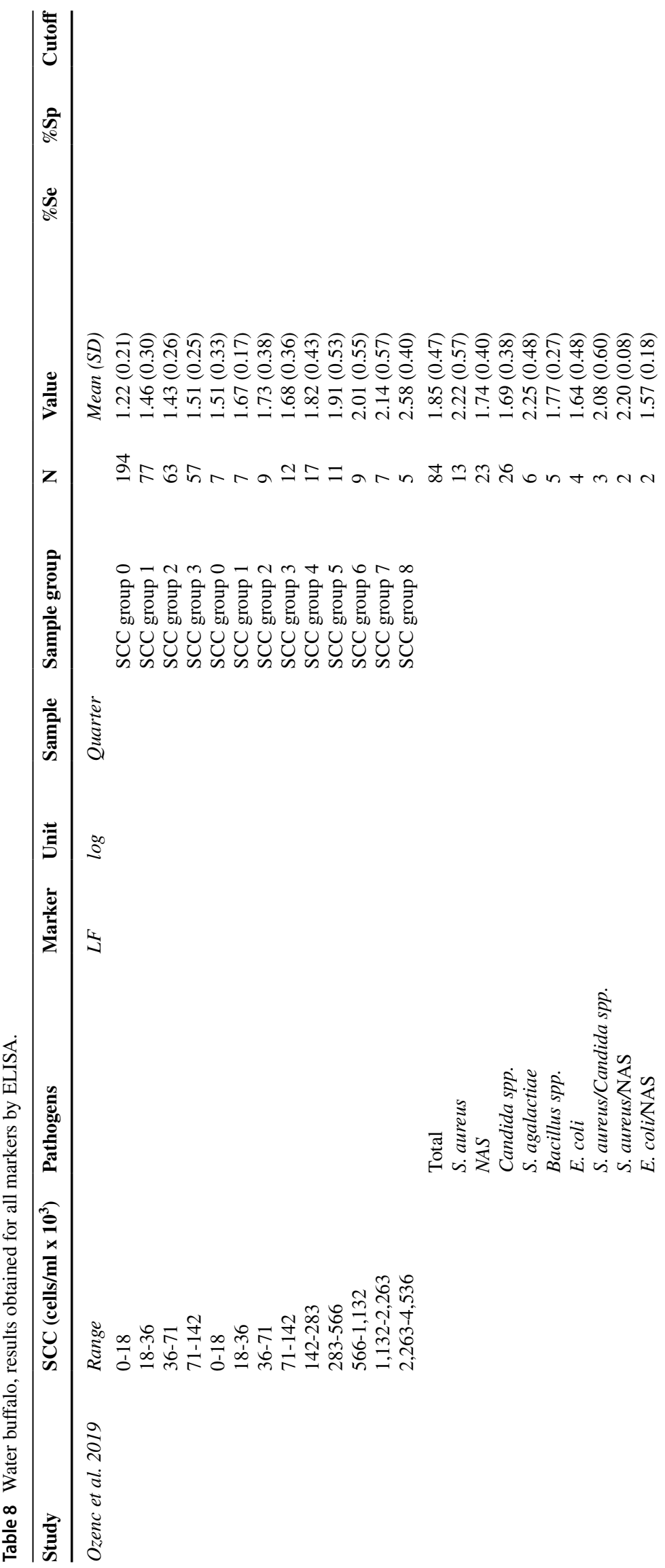

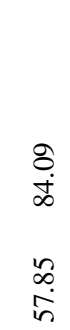

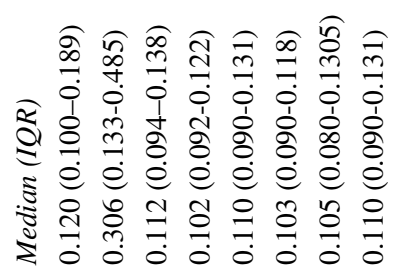

๗ิก

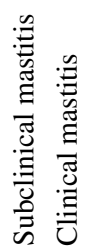

$\frac{0}{0}$

$\frac{8}{8}$

U

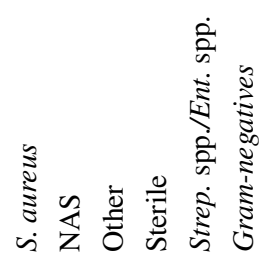

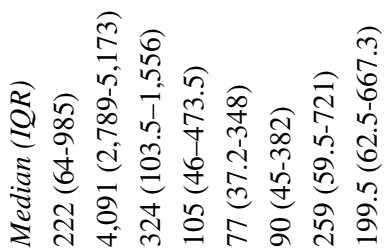

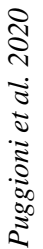


Concerning the risk of bias (Supplementary Table IX), on animal selection (domain 1) 18/33 (54.5\%) studies had a low risk of bias, $15 / 33(45.5 \%)$ high, and 0/33 (0.0\%) unclear risk. Regarding the index test (domain 2), one study out of $33(3.0 \%)$ had a low risk of bias, $29 / 33(87.9 \%)$ had high risk, and 3/33 (9.1\%) unclear risk. For the reference standard (domain 3), we observed a low risk of bias in 22/33 studies $(66.7 \%)$, high risk in $9 / 33(27.3 \%)$ and unclear risk in $2 / 33$ (6.1\%). Finally, flow and timing (domain 4) showed low risk of bias in 21/33 records (63.6\%), high risk in 11/33 (33.3\%), and unclear risk in 1/33 (3.0\%). Many studies showed low concerns about applicability, especially regarding domain 3 (Supplementary Table X) In detail, in domain 1, low risk was reported in $29 / 33(87.9 \%)$, high in $4 / 33(12.1 \%)$, and unclear in $0 / 53(0.0 \%)$. In domain 2 , records had low risk in $25 / 33(75.8 \%)$, high in $5 / 33(15.1 \%)$ and unclear $3 / 33$ (9.1\%); whereas in domain 3 we observed low risk in $31 / 33$ (93.9\%) papers, high in $2 / 33(6.1 \%)$ and unclear in $0 / 33$ $(0.0 \%)$.

\section{Conclusions and recommendations}

Our work aimed at analytically assessing the scientific literature describing the use of non-enzymatic milk proteins as mastitis markers in dairy ruminant species with the PRISMA approach. Moreover, we aimed at summarizing and comparing the diagnostic performances of the immunoassays developed for their detection in the milk. As expected, the most frequently mentioned biomarkers were M-SAA, HP, CATH, and LF, which were investigated both in experimental/ observational studies and in discovery/implementation approaches. Nonetheless, we observed several critical issues in study designs, reference standard methods (the lack of "gold standard"), index test (frequently performed without a blind approach), heterogeneity in the unit of measurement used for detecting the same biomarker, and the different type of statistical analysis performed, resulting in a heterogeneity of the collected data that was not amenable to meta-analysis. Unfortunately, this is a common finding in many meta-analyses and illustrates how important it is for case definitions and other criteria to be standardized between studies. Nevertheless, being related to the nature of the disease, some of these issues could hardly be solved, even because a truly reliable, sensitive, and specific reference diagnostic test does not exist. To deal with this, we applied an alternative synthesis method newly used in systematic reviews, the "Synthesis Without Meta-analysis" (SWiM), which improves transparency in reporting. The critical issues we observed further highlight the importance of title writing and keyword definition, both in the publishing and searching phases. When drafting these crucial parts of their manuscripts, using appropriate consensus terminology will maximize retrieval in bibliographic searches, enhancing article visibility and data usability.
Supplementary Information The online version contains supplementary material available at https://doi.org/10.1007/s11259-022-09901-y.

Acknowledgements The authors thank Dr. Eugenia Agnese Cannas for providing critical support and suggestions.

Availability of data and material The data used for the systematic review are presented in supplementary tables.

Code availability Not applicable

Authors' contributions AG: conceptualization, data analysis, data editing, manuscript drafting. MP: data analysis, manuscript drafting. ST: data analysis, manuscript drafting. SD: conceptualization, data analysis, data editing, manuscript drafting and revision. MFA: conceptualization, data analysis, data editing, manuscript drafting and revision.

Funding information This work was supported by a Ph.D. fellowship from the Istituto Zooprofilattico Sperimentale della Sardegna, in collaboration with the University of Sassari, to A.G., and by a Ph.D. fellowship from the University of Milan, to M.P.

\section{Declaration}

Conflicts of interest/Competing interests The authors declare no competing interests

Ethics approval Not applicable as animals were not involved in this study. The data reported here have been retrieved from the scientific literature.

Consent to participate Not applicable. The data reported here have been retrieved from the scientific literature.

Consent for publication Not applicable. The data reported here have been retrieved from the scientific literature.

Open Access This article is licensed under a Creative Commons Attribution 4.0 International License, which permits use, sharing, adaptation, distribution and reproduction in any medium or format, as long as you give appropriate credit to the original author(s) and the source, provide a link to the Creative Commons licence, and indicate if changes were made. The images or other third party material in this article are included in the article's Creative Commons licence, unless indicated otherwise in a credit line to the material. If material is not included in the article's Creative Commons licence and your intended use is not permitted by statutory regulation or exceeds the permitted use, you will need to obtain permission directly from the copyright holder. To view a copy of this licence, visit http://creativecommons.org/licenses/by/4.0/.

\section{References}

Addis MF, Pisanu S, Marogna G, Cubeddu T, Pagnozzi D, Cacciotto C, Campesi F, Schianchi G, Rocca S, Uzzau S (2013) Production and Release of Antimicrobial and Immune Defense Proteins by Mammary Epithelial Cells following Streptococcus uberis Infection of Sheep. Infect Immun 81:3182-3197. https://doi.org/10.1128/IAI.00291-13

Addis MF, Tedde V, Dore S, Pisanu S, Puggioni GMG, Roggio AM, Pagnozzi D, Lollai S, Cannas A, Uzzau S (2016a) Evaluation of milk cathelicidin for detection of dairy sheep mastitis. J Dairy Sci 99:6446-6456. https://doi.org/10.3168/jds.2015-10293 
Addis MF, Tedde V, Puggioni GMG, Pisanu S, Casula A, Locatelli C, Rota N, Bronzo V, Moroni P, Uzzau S (2016b) Evaluation of milk cathelicidin for detection of bovine mastitis. J Dairy Sci 99:8250-8258. https://doi.org/10.3168/jds.2016-11407

Addis MF, Bronzo V, Puggioni GMG, Cacciotto C, Tedde V, Pagnozzi D, Locatelli C, Casula A, Curone G, Uzzau S, Moroni P (2017) Relationship between milk cathelicidin abundance and microbiologic culture in clinical mastitis. J Dairy Sci 100:2944-2953. https://doi.org/10.3168/jds.2016-12110

Åkerstedt M, Waller KP, Sternesjö Å (2007) Haptoglobin and serum amyloid A in relation to the somatic cell count in quarter, cow composite and bulk tank milk samples. J Dairy Res 74:198-203. https://doi.org/10.1017/S0022029906002305

Åkerstedt M, Waller KP, Sternesjö Å (2009) Haptoglobin and serum amyloid A in bulk tank milk in relation to raw milk quality. J Dairy Res 76:483-489. https://doi.org/10.1017/S0022029909990185

Åkerstedt M, Forsbäck L, Larsen T, Svennersten-Sjaunja K (2011) Natural variation in biomarkers indicating mastitis in healthy cows. J Dairy Res 78:88-96. https://doi.org/10.1017/S0022029910000786

Bergonier D, de Crémoux R, Rupp R, Lagriffoul G, Berthelot X (2003) Mastitis in Dairy Small Ruminants. Vet Res 34:689-716. https:// doi.org/10.1051/vetres:2003030

Bochniarz M, Szczubiał M, Brodzki P, Krakowski L, Dąbrowski R (2020) Serum amyloid A as a marker of cow's mastitis caused by Streptococcus sp. Comp Immunol Microbiol Infect Dis 72:101498. https://doi.org/10.1016/j.cimid.2020.101498

Brenaut P, Lefèvre L, Rau A, Laloë D, Pisoni G, Moroni P, Bevilacqua C, Martin P (2014) Contribution of mammary epithelial cells to the immune response during early stages of a bacterial infection to Staphylococcus aureus. Vet Res 45:16. https://doi.org/10.1186/ 1297-9716-45-16

Campbell M, McKenzie JE, Sowden A, Katikireddi SV, Brennan SE, Ellis S, Hartmann-Boyce J, Ryan R, Shepperd S, Thomas J, Welch V (2020) Thomson H (2020) Synthesis without metaanalysis (SWiM) in systematic reviews: reporting guideline. BMJ 368:16890. https://doi.org/10.1136/bmj.16890

Chakraborty S, Dhama K, Tiwari R, Iqbal Yatoo M, Khurana SK, Khandia R, Munjal A, Munuswamy P, Kumar MA, Singh M, Singh R, Gupta VK (2019) Chaicumpa W (2019) Technological interventions and advances in the diagnosis of intramammary infections in animals with emphasis on bovine population-a review. Vet Q 39(1):76-94. https://doi.org/10.1080/01652176. 2019.1642546

Chen PW, Chen WC, Mao FC (2004) Increase of lactoferrin concentration in mastitic goat milk. J Vet Med Sci 66:345-350. https://doi. org/10.1292/jvms.66.345

Chen PW, Mao FC (2004) Detection of lactoferrin in bovine and goat milk by enzyme-linked immunosorbent assay. J Food Drug Anal 12:133-139. https://doi.org/10.38212/2224-6614.2653

Cheng JB, Wang JQ, Bu DP, Liu GL, Zhang CG, Wei HY, Zhou LY, Wang JZ (2008) Factors affecting the lactoferrin concentration in bovine milk. J Dairy Sci 91:970-976. https://doi.org/10.3168/ jds.2007-0689

Contreras A, Sierra D, Sánchez A, Corrales JC, Marco JC, Paape MJ, Gonzalo C (2007) Mastitis in small ruminants. Small Rum Res 68:145-153. https://doi.org/10.1016/j.smallrumres.2006.09.011

Cubeddu T, Cacciotto C, Pisanu S, Tedde V, Alberti A, Pittau M, Dore S, Cannas A, Uzzau S, Rocca S, Addis MF (2017) Cathelicidin production and release by mammary epithelial cells during infectious mastitis. Vet Immunol Immunopathol 189:66-70. https:// doi.org/10.1016/j.vetimm.2017.06.002

Dalanezi FM, Schmidt EMS, Joaquim SF, Guimarães FF, Guerra ST, Lopes BC, Cerri RLA, Chadwick C, Langoni H (2020) Concentrations of Acute-Phase Proteins in Milk from Cows with Clinical Mastitis Caused by Different Pathogens. Pathogens 9:706. https:// doi.org/10.3390/pathogens9090706
Eckersall PD, Young FJ, Nolan AM, Knight CH, McComb C, Waterston MM, Hogarth CJ, Scott EM, Fitzpatrick JL (2006) Acute phase proteins in bovine milk in an experimental model of Staphylococcus aureus subclinical mastitis. J Dairy Sci 89:14881501. https://doi.org/10.3168/jds.S0022-0302(06)72216-0

Ezzat Alnakip M, Quintela-Baluja M, Böhme K, Fernández-No I, Caamaño-Antelo S, Calo-Mata P, Barros-Velázquez J (2014) The immunology of mammary gland of dairy ruminants between healthy and inflammatory conditions. J Vet Med 2014:659801. https://doi.org/10.1155/2014/659801

Fetherston CM, Wells JI, Hartmann PE (2006) Severity of mastitis symptoms as a predictor of C-reactive protein in milk and blood during lactation. Breastfeeding Med 1:127-135. https://doi.org/ 10.1089/bfm.2006.1.127

Galfi AL, Radinović MZ, Boboš SF, Pajić MJ, Savić SS, Milanov DS (2016a) Lactoferrin concentrations in bovine milk during involution of the mammary glands, with different bacteriological findings. Vet Arhiv 86:487-497

Galfi AL, Radinović MZ, Milanov DS, Savić SS, Boboš SF, Pajić MJ (2016b) Lactoferrin and immunoglobulin $G$ concentration in bovine milk from cows with subclinical mastitis during the late lactation period. Acta Sci Vet 44:6. https://doi.org/10.22456/ 1679-9216.81097

Gerardi G, Bernardini D, Elia CA, Ferrari V, Iob L, Segato S (2009) Use of serum amyloid A and milk amyloid A in the diagnosis of subclinical mastitis in dairy cows. J Dairy Res 76:411-417. https://doi.org/10.1017/S0022029909990057

Grönlund U, Hultén C, Eckersall PD, Hogarth C, Persson Waller K (2003) Haptoglobin and serum amyloid A in milk and serum during acute and chronic experimentally induced Staphylococcus aureus mastitis. J Dairy Res 70:379-386. https://doi.org/10. 1017/s0022029903006484

Grönlund U, Hallén Sandgren C, Persson Waller K (2005) Haptoglobin and serum amyloid A in milk from dairy cows with chronic sub-clinical mastitis. Vet Res 36:191-198. https://doi. org/10.1051/vetres:2004063

Gulbe G, Pilmane M, Saulīte V, Doniṇa S, Jermolajevs J, Peškova L, Valdovskaet A (2020) Cells and cytokines in milk of subclinically infected bovine mammary glands after the use of immunomodulatory composition GLP 810. Mediators Inflamm 2020:8238029. https://doi.org/10.1155/2020/8238029

Hiss S, Mielenz M, Bruckmaier RM, Sauerwein H (2004) Haptoglobin concentrations in blood and milk after endotoxin challenge and quantification of mammary $\mathrm{Hp}$ mRNA expression. J Dairy Sci 87:3778-3784. https://doi.org/10.3168/jds.S00220302(04)73516-X

Hiss S, Mueller U, Neu-Zahren A, Sauerwein H (2007) Haptoglobin and lactate dehydrogenase measurements in milk for the identification of subclinically diseased udder quarters. Veterinarni Medicina 52:245-252. https://doi.org/10.17221/1879-VETMED

Hughes K, Watson CJ (2018) The mammary microenvironment in mastitis in humans, dairy ruminants, rabbits and rodents: a one health focus. J Mammary Gland Biol Neoplasia 23:27-41. https://doi.org/10.1007/s10911-018-9395-1

Hussein HA, El-Razik KAEA, Gomaa AM, Elbayoumy MK, Abdelrahman KA, Hosein HI (2018) Milk amyloid A as a biomarker for diagnosis of subclinical mastitis in cattle. Vet World 11:34 41. https://doi.org/10.14202/vetworld.2018.34-41

Iliev PT, Georgieva TM (2019) Acute phase biomarkers of diseases in small ruminants: an overview. Bulg J Vet Med 22:1-12. https://doi.org/10.15547/10.15547/bjvm.1051

Jaeger S, Virchow F, Torgerson PR, Bischoff M, Biner B, Hartnack S, Rüegg SR (2017) Test characteristics of milk amyloid A ELISA, somatic cell count, and bacteriological culture for detection of intramammary pathogens that cause subclinical mastitis. J Dairy Sci 100:7419-7426. https://doi.org/10.3168/jds.2016-12446 
Kelly AL, Leitner G, Merin U (2018) Milk Quality and Udder Health: Test Methods and Standards. Reference Module in Food Science, Elsevier, 2018, ISBN 9780081005965, doi: https://doi. org/10.1016/B978-0-08-100596-5.00951-3.

Kościuczuk EM, Lisowski P, Jarczak J, Strzałkowska N, Jóźwik A, Horbańczuk J, Krzyżewski J, Zwierzchowski L, Bagnicka E (2012) Cathelicidins: family of antimicrobial peptides. A review. Mol Biol Rep 39:10957-10970. https://doi.org/10.1007/ s11033-012-1997-x

Kováč G, Popelková M, Tkáčiková L, Burdová O, Ihnát O (2007) Interrelationship between somatic cell count and acute phase proteins in serum and milk of dairy cows. Acta Vet Brno 76:5157. https://doi.org/10.2754/avb200776010051

Kovačević-Filipović M, Ilić V, Vujčić Z, Dojnov B, Stevanov-Pavlović M, Mijačević Z, Božić T (2012) Serum amyloid A isoforms in serum and milk from cows with Staphylococcus aureus subclinical mastitis. Vet Immunol Immunopathol 145:120-128. https://doi. org/10.1016/j.vetimm.2011.10.015

Lam TJGM, Olde Riekerink RGM, Sampimon OC, Smith H (2009) Mastitis diagnostics and performance monitoring: a practical approach. Ir Vet J 62:S34-S39. https://doi.org/10.1186/ 2046-0481-62-S4-S34

Larson MA, Weber A, Weber AT, McDonald TL (2005) Differential expression and secretion of bovine serum amyloid A3 (SAA3) by mammary epithelial cells stimulated with prolactin or lipopolysaccharide. Vet Immunol Immunopathol 107:255-264. https://doi. org/10.1016/j.vetimm.2005.05.006

Lash JA, Coates TD, Lafuze J, Baehner RL, Boxer LA (1983) Plasma lactoferrin reflects granulocyte activation in vivo. Blood 61:885888. https://doi.org/10.1182/blood.V61.5.885.885

Leitner G, Shoshani E, Krifucks O, Chaffer M, Saran A (2000) Milk leucocyte population patterns in bovine udder infection of different aetiology. J Vet Med B Infect Dis Vet Public Health 47:581589. https://doi.org/10.1046/j.1439-0450.2000.00388.x

Lopez-Benavides MG, Dohoo I, Scholl D, Middleton J, Perez R (2012) Interpreting Bacteriological Culture Results to Diagnose Bovine Intramammary Infections. National Mastitis Council Research Committee Report

McDonald TL, Larson MA, Mack DR, Weber A (2001) Elevated extrahepatic expression and secretion of mammary-associated serum amyloid A 3 (M-SAA3) into colostrum. Vet Immunol Immunopathol 83:203-211. https://doi.org/10.1016/S0165-2427(01)00380-4

Menzies PI, Ramanoon SZ (2001) Mastitis of Sheep and Goats. Vet Clin North Am Food Anim Pract 17:333-358. https://doi.org/10. 1016/S0749-0720(15)30032-3

Miglio A, Moscati L, Fruganti G, Pela M, Scoccia E, Valiani A, Maresca C (2013) Use of milk amyloid A in the diagnosis of subclinical mastitis in dairy ewes. J Dairy Res 80:496-502. https:// doi.org/10.1017/S0022029913000484

Moher D, Liberati A, Tetzlaff J, Altman DG (2009) Preferred Reporting Items for Systematic Reviews and Meta-Analyses: The PRISMA Statement. PLoS Med 6:e1000097. https://doi.org/10.1371/journ al.pmed.1000097

Oliver SP, Gonzales RN, Hogan JS, Jayarao BM, Owens WE, Owens W (2004). Microbiological procedures for the diagnosis of bovine udder infection and determination of milk quality. $4^{\text {th }}$ Edition. National Mastitis Council, Inc. Verona (WI), USA.

Özenç E, Şeker E, Acar D, Koca HB, Yazıcı E, Çelik H, Doğan N, Avcı G, Yılmaz O, Küçükkebapçı M, Uçar M, Baştan A (2019) Milk lactoferrin concentrations in anatolian buffaloes with and without subclinical mastitis. Buffalo Bull 38:2941-2298

Pedersen LH, Aalbaek B, Røntved CM, Ingvartsen KL, Sorensen NS, Heegaard PM, Jensen HE (2003) Early pathogenesis and inflammatory response in experimental bovine mastitis due to Streptococcus uberis. J Comp Pathol 128:156-164. https://doi.org/10. 1053/jcpa.2002.0620
Persson Y, Olofsson I (2011) Direct and indirect measurement of somatic cell count as indicator of intramammary infection in dairy goats. Acta Vet Scand 53:15. https://doi.org/10.1186/ 1751-0147-53-15

Pisanu S, Cacciotto C, Pagnozzi D, Uzzau S, Pollera C, Penati M, Bronzo V, Addis MF (2020) Impact of Staphylococcus aureus infection on the late lactation goat milk proteome: new perspectives for monitoring and understanding mastitis in dairy goats. J Proteomics 221:103763. https://doi.org/10.1016/j.jprot.2020. 10376

Puggioni GMG, Tedde V, Uzzau S, Guccione J, Ciaramella P, Pollera C, Moroni P, Bronzo V, Addis MF (2020a) Evaluation of a bovine cathelicidin ELISA for detecting mastitis in the dairy buffalo: comparison with milk somatic cell count and bacteriological culture. Res Vet Sci 128:129-134. https://doi.org/10.1016/j.rvsc. 2019.11.009

Puggioni GMG, Tedde V, Uzzau S, Dore S, Liciardi M, Cannas EA, Pollera C, Moroni P, Bronzo V, Addis MF (2020b) Relationship of late lactation milk somatic cell count and cathelicidin with intramammary infection in small ruminants. Pathogens 9:37. https://doi.org/10.3390/pathogens9010037

Pyörälä S, Hovinen M, Simojoki H, Fitzpatrick J, Eckersall PD, Orro T (2011) Acute phase proteins in milk in naturally acquired bovine mastitis caused by different pathogens. Vet Rec 168:535-535. https://doi.org/10.1136/vr.d1120

Rossi RS, Amarante AF, Correia LBN, Guerra ST, Nobrega DB, Latosinski GS, Rossi BF, Rall VLM, Pantoja JCF (2018) Diagnostic accuracy of Somaticell, California Mastitis Test, and microbiological examination of composite milk to detect Streptococcus agalactiae intramammary infections. J Dairy Sci 101:10220 10229. https://doi.org/10.3168/jds.2018-14753

Ruegg PL (2017) A 100-Year Review: Mastitis detection, management, and prevention. J Dairy Sci 100:10381-10397. https://doi.org/10. 3168/jds.2017-13023

Safi S, Khoshvaghti A, Jafarzadeh SR, Bolourchi M, Nowrouzian I (2009) Acute phase proteins in the diagnosis of bovine subclinical mastitis. Vet Clin Pathol 38:471-476. https://doi.org/10.1111/j. 1939-165X.2009.00156.X

Sakemi Y, Tamura Y, Hagiwara K (2011) Interleukin-6 in quarter milk as a further prediction marker for bovine subclinical mastitis. J Dairy Res 78:118-121. https://doi.org/10.1017/S0022029910000828

Sargeant JM, O’Connor AM (2020) Scoping Reviews, Systematic Reviews, and Meta-Analysis: Applications in Veterinary Medicine. Front Vet Sci 7:11. https://doi.org/10.3389/fvets.2020.00011

Shaheen T, Bilal Ahmad S, Rehman MU, Muzamil S, Bhat RR, Hussain I, Bashir N, Mir MUR, Paray BA, Dawood MAO (2020) Investigations on cytokines and proteins in lactating cows with and without naturally occurring mastitis. Journal of King Saud University - Science 32:2863-2867. https://doi.org/10.1016/j. jksus.2020.07.009

Shimazaki K, Kawai K (2017) Advances in lactoferrin research concerning bovine mastitis. Biochem Cell Biol 95:69-75. https://doi. org/10.1139/bcb-2016-0044

Shirazi-Beheshtiha SH, Safi S, Rabbani V, Bolourchi M, Ameri M, Khansari MR (2011) The diagnostic value of determination of positive and negative acute phase proteins in milk from dairy cows with subclinical mastitis. Comp Clin Pathol 21:999-1003. https:// doi.org/10.1007/s00580-011-1216-5

Smolenski GA, Wieliczko RJ, Pryor SM et al (2011) The abundance of milk cathelicidin proteins during bovine mastitis. Vet Immunol Immunopathol 143:125-130. https://doi.org/10.1016/j.vetimm. 2011.06.034

Sobczuk-Szul M, Wielgosz-Groth Z, Nogalski Z, PogorzelskaPrzybyłek P (2014) Changes in the content of whey proteins during lactation in cow's milk with a different somatic cells count. Vet Med Zoot 65(87) 
Sordillo LM, Streicher KL (2002) Mammary gland immunity and mastitis susceptibility. J Mammary Gland Biol Neoplasia 7:135-146. https://doi.org/10.1023/a:1020347818725

Souza FN, Blagitz MG, Penna CFAM, Della Libera AMMP, Heinemann MB, Cerqueira MMOP (2012) Somatic cell count in small ruminants: Friend or foe? Small Rum Res 107:65-75. https://doi. org/10.1016/j.smallrumres.2012.04.005

Suojala L, Orro T, Järvinen H, Saatsi J, Pyörälä S (2008) Acute phase response in two consecutive experimentally induced E. coli intramammary infections in dairy cows. Acta Vet Scand 50(18). https://doi.org/10.1186/1751-0147-50-18

Szczubiał M, Dąbrowski R, Kankofer M, Bochniarz M, Komar M (2012) Concentration of serum amyloid A and ceruloplasmin activity in milk from cows with subclinical mastitis caused by different pathogens. Pol J Vet Sci 15:291-296. https://doi.org/10. 2478/v10181-011-0149-x

Tedde V, Bronzo V, Puggioni GMG, Pollera C, Casula A, Curone G, Moroni P, Uzzau S, Addis MF (2019) Milk cathelicidin and somatic cell counts in dairy goats along the course of lactation. J Dairy Res 86:217-221. https://doi.org/10.1017/S002202991 9000335

Thomas FC, Waterston M, Hastie P, Parkin T, Haining H, Eckersall PD (2015) The major acute phase proteins of bovine milk in a commercial dairy herd. BMC Vet Res 11:207. https://doi.org/10. 1186/s12917-015-0533-3

Tuaillon E, Viljoen J, Dujols P, Cambonie G, Rubbo PA, Nagot N, Bland RM, Badiou S, Newell ML, Van de Perre P (2017) Subclinical mastitis occurs frequently in association with dramatic changes in inflammatory/anti-inflammatory breast milk components. Pediatr Res 81:556-564. https://doi.org/10. 1038/pr.2016.220

van Harten RM, van Woudenbergh E, van Dijk A, Haagsman HP (2018) Cathelicidins: Immunomodulatory Antimicrobials. Vaccine (Basel) 6:63. https://doi.org/10.3390/vaccines60 30063
Viguier C, Arora S, Gilmartin N, Welbeck K, O'Kennedy R (2009) Mastitis detection: current trends and future perspectives. Trends in Biotechnol 27:486-493. https://doi.org/10.1016/j.tibtech.2009. 05.004

Welty FK, Larry Smith K, Schanbacher FL (1976) Lactoferrin Concentration During Involution of the Bovine Mammary Gland. J Dairy Sci 59:224-231. https://doi.org/10.3168/jds.S0022-0302(76) 84188-4

Wenz JR, Fox LK, Muller FJ, Rinaldi M, Zeng R, Bannerman DD (2010) Factors associated with concentrations of select cytokine and acute phase proteins in dairy cows with naturally occurring clinical mastitis. J Dairy Sci 93:2458-2470. https://doi.org/10. 3168/jds.2009-2819

Winter P, Colditz IG (2002) Immunological responses of the lactating ovine udder following experimental challenge with Staphylococcus epidermidis. Vet Immunol Immunopathol 89:57-65. https:// doi.org/10.1016/s0165-2427(02)00184-8

Wollowski L, Heuwieser W, Kossatz A, Addis MF, Puggioni GMG, Meriaux L, Bertulat S (2021) The value of the biomarkers cathelicidin, milk amyloid $\mathrm{A}$, and haptoglobin to diagnose and classify clinical and subclinical mastitis. J Dairy Sci 104:2106-2122. https://doi.org/10.3168/jds.2020-18539

Zanetti M (2004) Cathelicidins, multifunctional peptides of the innate immunity. J Leukocyte Biol 75:39-48. https://doi.org/10.1189/ jlb.0403147

Zanetti M (2005) The role of cathelicidins in the innate host defenses of mammals. Curr Issues Mol Biol 7:179-196

Zeng R, Bequette BJ, Vinyard BT, Bannerman DD (2009) Determination of milk and blood concentrations of lipopolysaccharidebinding protein in cows with naturally acquired subclinical and clinical mastitis. J Dairy Sci 92:980-989. https://doi.org/10.3168/ jds.2008-1636

Publisher's Note Springer Nature remains neutral with regard to jurisdictional claims in published maps and institutional affiliations. 